\title{
Pannexin 3 deletion reduces fat accumulation and inflammation in a sex-specific manner
}

\section{Affiliations:}

${ }^{1}$ Department of Anatomy and Cell Biology, Schulich School of Medicine and Dentistry, University of Western Ontario, London, Ontario, N6A 5C1, Canada

${ }^{5}$ Department of Physiology, University of Toronto, Toronto, Ontario, M5S 1A8, Canada

$14{ }^{6}$ Department of Physiology and Pharmacology, University of Western Ontario, London, Ontario,

${ }^{2}$ Western's Bone and Joint Institute, The Dr. Sandy Kirkley Centre for Musculoskeletal

${ }^{7}$ Robarts Research Institute, Schulich School of Medicine and Dentistry, University of Western

$18{ }^{8}$ Department of Chemical and Biomedical Engineering, University of Western Ontario, London,

$20{ }^{9}$ Department of Oncology, Division of Experimental Oncology, Schulich School of Medicine

21 and Dentistry, University of Western Ontario, London, Ontario, N6A 5C1, Canada

23 *To whom correspondence should be addressed: Silvia Penuela, PhD, Associate Professor,

24 Department of Anatomy and Cell Biology, University of Western Ontario, London, Ontario, 


\section{Abstract}

27 Background: Pannexin 3 (PANX3), is a channel-forming glycoprotein that enables nutrient-

28 induced inflammation in vitro, and genetic linkage data suggests it regulates body mass index.

29 Here, we characterized inflammatory and metabolic parameters in global Panx3 knockout (KO)

30 mice in the context of forced treadmill running (FEX) and high fat diet (HFD).

31 Methods: C57BL/6N (WT) and KO mice were randomized to either a FEX running protocol or

32 no running (SED) from 24 until 30 weeks of age. Body weight was measured biweekly, and body

33 composition was measured at 24 and 30 weeks of age. Male WT and KO mice were fed a HFD

34 from 12 - 28 weeks of age. Metabolic organs were analyzed for a panel of inflammatory markers

35 and PANX3 expression.

36 Results: In females there were no significant differences in body composition between genotypes,

37 which could be due to the lack of PANX3 expression in female white adipose tissue, while male

38 KOs fed a chow diet had lower body weight, and lower fat mass at 24 and 30 weeks of age, which

39 was reduced to the same extent as 6 weeks of FEX in WT mice. Additionally, male KO mice

40 exhibited significantly lower expression of multiple pro-inflammatory genes in white adipose

41 tissue compared to WT mice. While on a HFD body weight differences were insignificant, in KO

42 mice, multiple inflammatory genes were significantly differently expressed in quadriceps muscle

43 and white adipose tissue resulting in a more anti-inflammatory phenotype compared to WT mice.

44 The lower fat mass in male $\mathrm{KO}$ mice may be due to significantly fewer adipocytes in their

45 subcutaneous fat compared to WT mice. Mechanistically, adipose stromal cells (ASCs) cultured

46 from KO mice grow significantly slower than WT ASCs. Conclusion: PANX3 is expressed in

47 male adult mouse adipose tissue and may regulate adipocyte numbers, influencing fat

48 accumulation and inflammation. 


\section{Introduction}

50 Obesity is caused by excessive fat accumulation and is a major contributor to many co-morbidities

51 including type II diabetes and cardiovascular disease (1). While exercise training and caloric

52 deficit are effective treatments for obesity, many find these interventions difficult to implement

53 and sustain (2). Genetic factors increase susceptibility to weight gain (3), and understanding which

54 genetic factors underlie obesity will assist clinicians in determining the best pharmacotherapeutic

55 options for a given patient.

56 Pannexins (PANX1, 2, 3) are channel-forming glycoproteins that allow the passage of ions and

57 metabolites for autocrine and paracrine signaling in a variety of cells (4). Previous reports have

58 shown that PANX1 is expressed in adipocytes and has a functional role in immune cell recruitment

59 (5), adipocyte hypertrophy and fat accumulation (6), glucose metabolism (7), and

60 thermoregulation in brown fat (8). Recent evidence suggests that PANX3 may also play a role in

61 adipogenesis and inflammation (9-11). Using a systems approach involving quantitative trait loci

62 mapping and gene expression network analysis, Halliwill and colleagues found that the Panx3

63 gene is linked to body mass index in male mice (9). This group also identified Panx3 as a

64 component of the homeodomain-interacting protein kinase 2 (Hipk2) gene network which is

65 involved in adipocyte signaling and differentiation (10). These studies provide indirect evidence

66 that PANX3 may be involved in the molecular mechanism of fat accumulation.

67 A consequence of excessive fat accumulation is inflammation of adipose tissue (12-15). This

68 inflammation is thought to contribute to many comorbidities $(14,16-19)$. We previously reported

69 that the saturated-fatty acid palmitate activated cell-intrinsic pro-inflammatory programs in

70 isolated muscle cells and concomitantly increased Panx3 expression (11). Additionally, we

71 demonstrated that PANX3 channels allowed adenosine triphosphate (ATP) release, attracting 
72 monocytes towards the muscle cells (11). This would suggest that PANX3 may be a contributor

73 to nutrient-induced skeletal muscle inflammation by acting as a conduit for 'find me' signals to

74 immune cells. Lastly, we observed that HFD induced the expression of Panx3 in adipose tissue

75 (11), which was the first published finding of $\operatorname{Panx3}$ expression in mouse adipose tissue. However,

76 its role in diet-induced obesity, fat accumulation, inflammation and metabolism has not been

77 investigated.

78 Considering the evidence above, we sought out to determine the physiological effects of a global

79 deletion of Panx3 in mice exposed to forced exercise (FEX) and dietary excess (HFD). In males,

80 Panx3 Knockout (KO) mice had lower body weight and fat mass, but higher lean mass corrected

81 for body weight, and lower inflammation in adipose and quadriceps tissue compared to WT mice

82 to the same extent as 6 weeks of forced treadmill running. This potentially beneficial loss of natural

83 inflammatory gene expression level was still evident when challenged with caloric excess.

84 However, there were minimal differences between female WT and KO mice, highlighting a sex-

85 specific effect of the Panx3 deletion. This would suggest that the deletion of Panx3 attenuates fat

86 accumulation and inflammation in males and could become a useful, sex-specific, genetic target

87 to combat obesity and its associated inflammation.

\section{Materials and Methods}

89 Animals and ethics

90 Experiments performed on animals were approved by the Animal Care Committee of the

91 University Council on Animal Care at the University of Western Ontario, London ON, Canada

92 (UWO \# 2019-069), and in accordance with relevant guidelines and regulations. Panx3 KO mice

93 were generated as described previously (20). Panx3 KO mice were backcrossed with C57BL/6 N 
94 mice from Charles River Canada (Saint-Constant, PQ) until a congenic line was obtained

95 (minimum of 10 backcrossed generations). Mice were weaned at 3 weeks of age and fed either a

96 chow diet $(6.2 \%$ kcals from fat), Western (45\% kcals from fat) or a HFD (60\% kcals from fat, Test

97 Diet 58Y1) as described in the respective sections. At termination, mice were sacrificed using

98 carbon dioxide. Immediately after, blood was collected via cardiac puncture, adipose, quadriceps,

99 and liver tissues were collected, immediately snap frozen and stored at $-80^{\circ} \mathrm{C}$.

100 Forced exercise (FEX) protocol

101 At 24 weeks of age (baseline), mice were randomized to either sedentary (SED) or FEX groups.

102 The FEX groups were forced to run on a treadmill (Columbus Instruments, Ohio) for 6 weeks, 1

103 hour a day, 5 days a week, at a speed of $11 \mathrm{~m} / \mathrm{s}$, and a $10^{\circ}$ incline. The mice were encouraged to

104 run using a bottle brush bristle and a shock grid at the end of the treadmill as per the animal ethics

105 protocol. Mice were acclimatized for 10 mins before each session, which consisted of being in the

106 treadmill with no belt movement.

\section{Body composition}

108 Fat and lean mass composition were measured at baseline and 30 weeks of age using a quantitative

109 magnetic resonance (echo-MRI) mobile unit (Avian Facility of Advanced Research, University of

110 Western Ontario, London, ON, Canada) as described previously (6). Measurements were taken in 111 triplicate to verify the results.

\section{Blood glucose tolerance and plasma analysis}


113 Mice were fasted 4 hours prior to testing. Fasted blood glucose was measured via a glucometer

114 (OneTouch Ultra). Glucose tolerance testing was conducted by administration of $1 \mathrm{~g} / \mathrm{kg}$ of glucose

115 by intraperitoneal injection, and blood glucose was monitored at $0,15,30,60$, and 120 minutes

116 via tail vein puncture. Glucose area under the curve (AUC) was calculated. At sacrifice, blood was

117 collected, and plasma was isolated. ELISAs (ALPCO, NH, USA) were performed following the

118 manufacturer's protocol for insulin, cholesterol was assessed by CHOD-PAP kit (Roche

119 Diagnostics, Indianapolis, IN), and triglyceride analysis was conducted by Triglycerol/Glycerol

120 kit (Roche Diagnostics, Indianapolis, IN) following manufacturer's protocols.

\section{Metabolic cage analysis}

122 Metabolic analysis was assessed using the Comprehensive Lab Animal Monitoring System

123 (CLAMS) with the Oxymax software (Columbus Instruments, Columbus, OH, USA) at the

124 Robarts Research Institute. Mice were individually caged and acclimated for 24 hours prior to

125 measurement of food consumption, water consumption, energy expenditure, volume of oxygen

126 (VO2) and carbon dioxide (VCO2), respiratory exchange ratio (RER), total activity, total

127 ambulatory activity, and sleep duration, as described previously (6).

\section{RNA extractions, cDNA synthesis and qPCR}

129 Tissue RNA extraction were performed using total RNA isolation (TRIzol) reagent (Life

130 Technologies) and phenol-chloroform phase separation. Samples were homogenized in TRIzol,

131 mixed with chloroform and centrifuged at $12,000 \mathrm{xg}$ for 15 minutes at $4^{\circ} \mathrm{C}$. The aqueous phase

132 containing the RNA was isolated. Isopropanol was added and samples incubated at room 
133 temperature for 30 minutes to precipitate the RNA. The extracted RNA was pelleted by

134 centrifugation at $12,000 \mathrm{x}$ g for 10 minutes at $4^{\circ} \mathrm{C}$. The pellets were washed with $70 \%$ ethanol,

135 centrifuged at 7,500 x g for 5 minutes, and then again washed with $100 \%$ ethanol and centrifuged

136 lastly at 7,500 x g for 5 minutes. The samples were stored in $-80^{\circ} \mathrm{C}$.

137 NanoDrop 2000c spectrophotometer (NanoDrop) was used to quantify the extracted RNA

138 concentration and its purity. SuperScript variable input linear output (VILO) kit for

139 complementary deoxyribonucleic acid (cDNA) synthesis (Life Technologies) was used to

140 synthesize cDNA. cDNA synthesis reaction was performed in $10 \mu \mathrm{L}$ volume to which up to 125

$141 \mathrm{ng} / \mu \mathrm{L}$ RNA was added and a final concentration of $1 \mathrm{X}$ VILO Reaction mix and $1 \mathrm{X}$ SuperScript

142 Enzyme mix were loaded. The 96-well plate containing the samples were incubated at $42^{\circ} \mathrm{C}$ for

$14360 \mathrm{~min}$, then $85^{\circ} \mathrm{C}$ for $5 \mathrm{~min}$ on a $\mathrm{C} 1000$ thermal cycler (Bio-Rad), then stored at $-20^{\circ} \mathrm{C}$.

144 A reaction with $20 \mathrm{ng}$ of cDNA was used for each reverse transcriptase quantitative polymerase

145 chain reaction (RT-qPCR) along with 1X TaqMan Fast Advanced Master Mix, and predesigned

146 TaqMan probes (Life Technologies) for the following target genes: Arg1 (Mm00475988_m1),

147 Mrc1 (Mm00485148), IL-10 (Mm00439614), Chi313 (Mm00657889), Emr1

148 (Mm00802529_m1), IL-12a (Mm00434165), CCL2 (Mm00441242), Itgax (Mm00498701_m1),

149 Nos2 (Mm00440502_m1), and Tnf (Mm00443258_m1) on a StepOne Plus Real-Time PCR

150 System (Life Technologies). Samples were held at $95^{\circ} \mathrm{C}$ for 20 seconds, then cycled from $95^{\circ} \mathrm{C}$

151 for $1 \mathrm{~s}$ to $60^{\circ} \mathrm{C}$ for $20 \mathrm{~s}$ for 40 cycles. Gene expression of target genes were normalized to average

152 of the housekeeping genes Abt1 (Mm00803824_m1), Hprt (Mm03024075_m1), and/or Eef2

153 (Mm01171435 gH) using the $\Delta \Delta \mathrm{Ct}$ method. An inflammatory index score was calculated as the 
154 ratio of the sum of pro-inflammatory gene expression over the sum of the anti-inflammatory

155 gene expression and reflects the inflammatory status of the tissue.

156 For analysis of Panx3 mRNA expression in visceral fat tissue, RNA was extracted using a

157 combination of Trizol and a Qiagen RNeasy mini kit as was previously described (21). mPanx3

158 Forward: TTTCGCCCAGGAGTTCTCATC, Reverse: CCTGCCTGACACTGAAGTTG, m18S

159 Forward: GTAACCCGTTGAACCCCATT, Reverse: CCATCCAATCGGTAGTAGCG and

$160 \mathrm{mHprt}$. Normalized mRNA expression levels were analyzed using the $\Delta \Delta \mathrm{CT}$ method which was

161 calculated using BioRad CFX Manager Software. Aliquots were taken from the reactions, dyed

162 with ethidium bromide and electrophoresed on a $10 \%$ agarose gel.

\section{Protein analysis}

164 Protein lysates were extracted with lysis buffer containing: 1\% Triton X-100, $150 \mathrm{mM} \mathrm{NaCl}, 10$

165 mM Tris, 1 mM EDTA, 1 mM EGTA, 0.5\% NP-40 or a RIPA buffer (50 mM Tris-HCl pH 8.0,

$166150 \mathrm{mM} \mathrm{NaCl}, 1 \% \mathrm{NP}-40$ (Igepal), $0.5 \%$ sodium deoxycholate). Each buffer contained $1 \mathrm{mM}$

167 sodium fluoride, $1 \mathrm{mM}$ sodium orthovanadate, and half of a tablet of complete-mini EDTA-free

168 protease inhibitor (Roche, Mannheim, Germany). Protein was quantified by bicinchoninic acid

169 (BCA) assay (Thermo Fisher Scientific). Protein lysates (40 $\mu \mathrm{g})$ were separated by 10\% SDS-

170 PAGE and transferred onto a nitrocellulose membrane using an iBlotTM System (Invitrogen,

171 USA). Membranes were blocked with 3\% bovine serum albumin (BSA) with $0.05 \%$ Tween-20

172 in 1X phosphate buffer saline (PBS) and incubated with anti-mouse PANX3 antibody (1:1000;

173 PANX3 CT-379) (22), and anti-GAPDH antibody (1:1000; Millipore Cat\# MAB374). For

174 detection, IRDye ${ }^{\circledR}-800 \mathrm{CW}$ and -680RD (Life Technologies, USA) were used as secondary

175 antibodies at 1:10,000 dilutions and imaged using a LI-COR Odyssey infrared imaging system 
176 (LI-COR Biosciences, USA). Western blot quantification and analysis was conducted using

177 Image Studio ${ }^{\mathrm{TM}}$ Lite (LI-COR Biosciences). Positive controls were generated by ectopic

178 expression of PANX3 constructs in human embryonic kidney 293T (HEK293T) cells as

179 described before $(6,22)$.

180 Histological staining and subcutaneous adipocyte measurements

181 Dorsal skin samples from adult male WT and KO mice (12-months old) on a chow diet were

182 fixed in 10\% neutral buffered formalin and subsequently embedded in paraffin. Sections $(5 \mu \mathrm{m})$

183 were deparaffinized in xylene, rehydrated in graded alcohols, and washed in PBS. Parallel tissue

184 sections were stained with hematoxylin/eosin. Images were collected using a Leica DM IRE2

185 inverted epifluorescence microscope. Measurement of adipocyte cellular size (area) and number

186 of measured adipocytes was performed using the analytical software ImageJ (v.1.50i, National

187 Institute of Health, USA) by a blinded assessor. At least three tissue sections from each mouse

188 were analyzed and individual adipocytes with complete boundaries were selected for

189 quantification and counting.

\section{3T3-L1 cell culture and adipogenic induction}

191 Mouse embryonic fibroblast pre-adipocyte (3T3-L1) cells were purchased from ATCC and

192 checked for mycoplasma before use. Cells were grown in Dulbecco’s Modified Eagle's Medium

193 (DMEM) with $4.5 \mathrm{~g} / \mathrm{L}$ glucose, 1\% Pen-Strep, and 10\% calf serum (Thermo Fisher Scientific)

194 and cells below passage 10 were included in the studies. Adipogenic media for days 1-2

195 contained: DMEM with 4.5 g/L glucose (Thermo Fisher Scientific), 10\% calf serum (Thermo

196 Fisher Scientific), 1\% Pen-Strep, 100 $\mathrm{g} / \mathrm{mL}$ of isobutylmethylxanthine (IBMX), $390 \mathrm{ng} / \mathrm{mL}$

197 dexamethosone, and 5 $\mathrm{g} / \mathrm{mL}$ insulin (Sigma Aldrich). Adipogenic media for days 3-4 contained 
198 all of the above components without IBMX or dexamethasone. Following day 4, cells were fed

199 every 2-3 days with DMEM + 10\% FBS (Thermo fisher Scientific) until differentiation was

200 complete at day 10.

\section{Adipose-derived stromal cell isolation}

202 ASCs were isolated as described previously (6). WT and KO male mice were fed on the HFD,

203 with the modification of isolating cells from the inguinal adipose depot and cells were filtered

204 through a $100 \mu \mathrm{m}$ filter to remove debris prior to cell seeding. Fat from up to three mice was

205 pooled together for each separate isolation. Cells were seeded at high density $\left(80000 \mathrm{cells} / \mathrm{cm}^{2}\right)$

206 and rinsed 24 hours after isolation with sterile PBS and passaged when confluent (approximately

2077 days). ASCs were grown in DMEM: Ham’s F-12 (Sigma Aldrich), supplemented with 10\%

208 fetal bovine serum and 1\% Pen-Strep and growth medium was changed every 2 days. ASCs used

209 for assays were grown to Passage 2.

\section{Growth curves and adipogenic differentiation of ASCs}

211 ASCs from WT and Panx3 KO mice were plated in 12 well plates at a seeding density of 10000

212 cells $/ \mathrm{cm}^{2}$. Cell counts were measured in triplicate every other day up until day 7 using an

213 automated cell counter, Countess II (Thermo Fisher Scientific). Cells were fed every other day

214 with DMEM: Ham’s F12 media, 10\% FBS, 1\% Pen-Strep, (Sigma Aldrich). Adipogenic

215 induction was conducted with WT ASCs plated in 6 well plates at a seeding density of 30000

216 cell $/ \mathrm{cm}^{2}$. Adipogenic media as previously described (23) with the modifications of substituting

$2171 \mu \mathrm{g} / \mathrm{mL}$ Troglitazone and $0.25 \mathrm{mM}$ IBMX(Sigma Aldrich) for days 1-3. Media was changed

218 every other day for 14 days. 


\section{Statistical analysis}

220 Statistical analyses were performed using GraphPad Prism Version 9.20 (GraphPad, San Diego,

221 CA). Outliers were removed from data sets using the outlier test from GraphPad Prism Version

222 9.20. Normality tests were used to determine similar variation among the groups for fat mass in

223 males. A power analysis was conducted using the male baseline fat mass mean and standard

224 deviation data to determine an appropriate samples size for the 30-week time point analysis. Body

225 weight progression was analysed using a three-way repeated measures ANOVA with genotype $\mathrm{x}$

226 activity $\mathrm{x}$ age as factors. Single time point measures between genotypes were analysed using an

227 unpaired t-test. A two-way ANOVA with genotype $\mathrm{x}$ activity as factors was used for 30-week time

228 points, and other two-variable analyses. For blood glucose tolerance curves, a three-way factorial

229 ANOVA was used with genotype $\mathrm{x}$ activity $\mathrm{x}$ time as factors. Data are presented as

230 mean \pm standard error (SEM). $\mathrm{N}$ indicates number of animals.

\section{Results}

232 Male Panx3 KO mice weigh less, have less fat mass and more lean mass than WT mice to the 233 same extent as 6 weeks of forced exercise.

234 Male and female WT and KO congenic mice were bred, fed ad libitum on a normal rodent chow

235 diet, and randomly allocated to either a SED or FEX protocol from baseline to 30 weeks of age

236 (Fig. 1a). Body weights were tracked bi-weekly, and body composition and blood glucose

237 tolerance were analyzed at baseline and at 30 weeks of age. Postmortem, livers, skeletal muscle,

238 and visceral fat were collected for protein and mRNA analysis.

239 In males, KO mice weighed significantly less than WT mice as they aged (Fig. 1b). When

240 analyzing the baseline and 30-week body weights with and without exercise, KO mice weighed 
241 significantly less at baseline, and at 30 weeks compared to SED WT mice, but were not 242 significantly different from the FEX WTs as exercise attenuated weight gain in WT mice (Fig. 1c).

243 Considering that Panx3 may be involved in adipogenesis (10), is linked to body mass index (9),

244 and resulted in lower body weight in the present study, we then sought to determine if this lower

245 body weight in $\mathrm{KO}$ mice is due to differences in fat and/or lean mass. KO mice had significantly

246 less fat mass (Fig. 1d) and fat mass corrected for body weight (Fig. 1e) than WT mice at baseline.

247 At 30 weeks of age, SED and FEX KO mice had significantly lower fat mass (Fig. 1d) and fat

248 mass corrected for body weight (Fig. 1e) compared to SED WT mice. Interestingly, FEX

249 significantly decreased fat mass in WT mice, while FEX had no additional effect on fat mass in

250 KO mice (Fig. 1d \& e). This suggests that the deletion of Panx3 alone has a profound effect on fat

251 mass that is not further decreased by FEX. While there were no significant differences in raw lean

252 mass among the groups (Fig. 1f), when lean mass was corrected for body weight, KO mice had

253 significantly more lean mass compared to WT mice at baseline (Fig. 1g). Additionally, at 30 weeks

254 of age, SED KO mice had significantly higher lean mass corrected for body weight compared to

255 SED WT mice (Fig. 1g). However, the FEX WT mice had similar lean mass when corrected for

256 body weight compared to KO mice. These results suggest that the deletion of Panx3 reduces fat

257 mass and increases lean mass to the same extent as 6 weeks of FEX in male WT mice.

258 Using individual metabolic cage analysis, we found that there were no significant differences in

259 O2 Volume (Fig. S1a), CO2 Volume (Fig. S1b), Respiratory Exchange Ratio (Fig. S1c), Energy

260 Expenditure (Fig. S1d), Water Consumed (Fig. S1f), Total Activity (Fig. S1g), Ambulatory

261 Activity (Fig. S1h), or Sleep Time (Fig. S1i) between male WT and KO mice. However, there was

262 a main effect for Food Consumption suggesting that KO mice ate more food overall (Fig. S1e), 
263 indicating that the reduced body weight and fat mass in $\mathrm{KO}$ mice was not due to increased activity

264 or reduced food consumption.

265 Male Panx3 KO mice have lower inflammatory index in quadriceps and visceral fat tissues 266 compared to WT mice.

267 Changes in adiposity and lean mass could be correlated to inflammatory activation of adipose, 268 liver, and skeletal muscle tissue (24). Therefore, we next determined if Panx3 deletion influences 269 inflammatory gene expression in these metabolic tissues. Liver, quadriceps muscle and visceral 270 white adipose tissues were collected from male WT and KO mice from both SED and FEX groups 271 for analysis of inflammatory genes and an inflammatory index was calculated. Macrophage 272 markers Emrl and Itgax (CD11c), pro-inflammatory genes Tnfo, Nos2, Il12a, Ccl2 and Il6 and 273 anti-inflammatory markers $\mathrm{Arg} 1, \mathrm{Mrc1}$, IllO and Chi3l3 were analyzed in these tissues using RT-

274 qPCR. An inflammatory index was calculated as the ratio of the sum of the pro-inflammatory 275 markers over the sum of the anti-inflammatory markers and reflects the inflammatory status of the 276 tissue. In quadriceps, SED WT mice had significantly higher Emrl compared to all other groups, 277 while there was a main effect for KO mice having significantly higher Nos2, Tnf $\alpha$, and Il12a 278 compared to WT mice (Fig. 2a). When analyzing the inflammatory index for the quadriceps, no 279 group was significantly different than SED KO mice (Fig. 2b). In visceral adipose tissue, KO mice 280 had significantly lower pro-inflammatory markers Emrl, Itgax, Ccl2, Tnf $\alpha$, and anti-inflammatory 281 markers $\mathrm{Mrcl}$ (Fig. 2c). KO mice had significantly lower inflammatory indexes in visceral fat 282 compared to both SED and FEX WT mice (Fig. 2d). However, there were no significant 283 differences among the groups for any genes in the liver or the overall inflammatory index (Fig. 2e 
$284 \& \mathrm{f})$. These results suggest that the deletion of Panx3 results in a potential shift of inflammatory

285 tone in skeletal muscle and white adipose tissue, comparable to the effects of FEX.

286 Despite these changes in body composition and potential anti-inflammatory effect in skeletal 287 muscle and adipose tissue from a global deletion of Panx3, there were no significant differences

288 in blood glucose tolerance between genotypes (Fig. S2a, b, \& c). However, when analyzing the 289 blood glucose tolerance curves for the 30-week time point the p-value for genotype approached 290 significance $(\mathrm{p}=0.0528)$ (Fig. S2b). There were no significant differences in other circulating 291 measures of metabolic health such as insulin (Fig. S2d), cholesterol (Fig. S2e), and triglycerides 292 (Fig. S2f).

293 When analyzing circulating measures of inflammation, we found that SED KO mice had 294 significantly lower levels of total adiponectin compared to SED WT mice (Fig. S2g). However, 295 there were no significant differences in heavy molecular weight (HMW) adiponectin (Fig. S2h) or 296 the ratio of total/HMW adiponectin (Fig. S2i). Additionally, there were no differences in serum 297 amyloid A (SAA) between genotypes (Fig. S2j). However, there was a significant main effect for

298 Genotype suggesting KO mice have significantly lower circulating levels of IL-6 compared to WT 299 mice regardless of activity (Fig. S2k).

300 Female Panx3 KO mice weigh slightly less than WT mice with no significant differences in 301 body fat or lean mass.

302 To determine if these differences between genotypes are seen in females, we next compared WT 303 and KO female mice under both SED and FEX conditions. Interestingly in females, KO mice 304 weighed significantly less as they aged, indicated by a significant Genotype x Age interaction (p $305<0.0001$ ) (Fig. 3a). When analyzing baseline and 30-week-old data, there was a significant main 
306 effect for Genotype, suggesting female KO mice weighed significantly less than WT females,

307 however the effect size was much smaller than in males (Fig. 3b). Despite differences in body

308 weight, there were no significant differences between genotypes in body fat (Fig. 3c), body fat

309 corrected for body weight (Fig. 3d), lean mass (Fig. 3e), or lean mass corrected for body weight

310 (Fig. 3f) at baseline or with and without FEX at 30 weeks of age between genotypes, but there was

311 an effect for Activity in reducing fat mass regardless of genotype. This would suggest that Panx3's

312 role in fat accumulation in females is not as pronounced as in male mice.

313 Female Panx3 KO mice have higher inflammation in quadriceps and liver tissues compared

314 to WT mice.

315 Female WT and KO mice from both SED and FEX groups were sacrificed, and skeletal muscle,

316 visceral adipose, and liver tissues were excised for analysis as described above. In quadriceps,

317 FEX KOs had significantly higher expression of Nos2 expression compared to SED WT and KO

318 mice, while WT mice had significantly higher expression of Mrcl expression (Fig. 4a). KO mice

319 had significantly higher overall inflammatory index in quadriceps compared to WT mice (Fig. 4b).

320 In visceral fat, both SED and FEX KO mice had significantly lower expression of TNF $\alpha$ compared

321 to SED WT mice and significantly higher expression of Argl regardless of activity (Fig. 4c).

322 However, there were no significant differences among the groups when assessing the inflammatory

323 index for visceral fat (Fig. 4d). In liver, KO mice had significantly higher expression of $T N F \alpha$

324 regardless of activity group, and lower Argl and Chi313 compared to SED WT animals (Fig. 4e).

325 For the overall liver inflammatory index, $\mathrm{KO}$ mice had a significantly higher score compared to

326 WT mice regardless of activity (Fig. 4f). This would suggest that in female mice the deletion of

327 Panx3 leads to a higher inflammatory tone in the quadriceps and liver tissues. 
328 When assessing blood glucose tolerance in females, there was no significant difference at baseline

329 (Fig. S3a), however at the 30-week timepoint there was a significant Genotype x Activity

330 interaction (Fig. S3b). While there was no significant effect of exercise on AUC in KO mice, WT

331 mice seemed to have improved glucose handling with FEX (Fig. S3c). Additionally, there was an

332 overall main effect of Genotype for AUC, suggesting KO mice have improved glucose handling

333 (Fig. S3c). There were no significant Genotypic effects on insulin (Fig. S3d), triglycerides (Fig.

334 S3e), or cholesterol (Fig. S3f). However, there was a main effect of Activity for insulin (Fig. S3d).

335 When assessing circulating levels of inflammatory markers there were no significant differences

336 in total adiponectin (Fig. S3g), HMW adiponectin (Fig. S3h), total/HMW adiponectin (Fig. S3i),

337 SAA (Fig. S3j), and IL-6 (Fig. S3k).

338 PANX3 expression is higher in male visceral fat and is regulated by FEX and dietary caloric

339 excess.

340 Considering Panx3 deletion is producing sex differences in fat and lean mass, we next wanted to

341 determine if PANX3 expression is different between male and female visceral fat. Protein from

342 visceral fat of both SED and FEX male and female WT mice was isolated and ran on a Western

343 blot (Fig. 5a \& b). Males had significantly higher expression of PANX3 compared to females

344 regardless of activity levels. Interestingly, FEX seemed to increase PANX3 expression, but this

345 did not reach significance $(\mathrm{p}=0.0607)($ Fig. 5a). Next, considering Pillon et al. previously showed

346 that HFD significantly increases Panx3 mRNA expression in fat (11), we wanted to confirm these

347 results, and determine if FEX was able to counter this effect. Visceral fat mRNA was isolated from

348 male WT mice that ate either chow or a Western diet (45\% kcal from fat) and were subjected to

349 either SED or FEX conditions (Fig. 5c \& d). Western diet significantly increased Panx3 expression 
350 compared to chow fed animals, however, FEX attenuated this expression in Western fed animals

351 (Fig. 5c \& d). Next, we fed male WT and KO mice a HFD (60\% kcal from fat) from 12 to 28

352 weeks of age. Like Pillon et al. (11) and our mRNA results, HFD significantly increased PANX3

353 expression in fat compared to chow fed animals (Fig. 5e \& f). Next, we wanted to determine if

354 deleting Panx3 would have a protective effect on body weight under HFD feeding as seen in chow

355 fed male mice. However, there were no significant differences in raw body weight (Fig. 5g) or

356 body weight fold change (Fig. 5h) between WT and KO mice when on a HFD.

357 Male Panx3 KO mice fed a HFD have less inflammation in epidydimal adipose and skeletal

358 muscle tissue.

359 Considering HFD regulated PANX3 expression in adipose tissue, and PANX3 may mediate

360 nutrient-induced inflammation (11), we then set out to determine if $\mathrm{KO}$ mice are protected from

361 diet-induced inflammation. At sacrifice, HFD fed KO and WT mice had liver, quadriceps muscle

362 and epidydimal white adipose tissues (eWAT) collected for analysis of inflammatory markers, as

363 described above. KO mice had significantly higher expression of anti-inflammatory genes Argl

364 and IllO compared to WT mice (Fig. 6a), resulting in a significantly lower inflammatory index in

365 quadriceps (Fig. 6 b). In eWAT tissue (Fig. 6 c \& d) KO mice had lower expression of pro-

366 inflammatory genes Ccl2 and Il6, and significantly lower Argl and higher Chi313 anti-

367 inflammatory expression, resulting in significantly lower inflammatory index (Fig. 6d). However,

368 there were no significant differences in individual gene expression (Fig. 6e) or overall

369 inflammatory index in the liver (Fig. 6f) between genotypes. These results suggest that male KO

370 mice have lower skeletal muscle and fat tissue inflammatory tone compared to WT mice while on 371 an HFD. 


\section{Panx3 KO mice have fewer adipocytes, and their ASCs grow slower than WT mice.}

373 Considering we found that male KO mice have significantly lower fat mass than WT mice, we 374 wanted to determine if this was the result of less adipocytes or a reduction in adipocyte 375 hypertrophy. While there were no differences in the size of subcutaneous adipocytes between KO 376 and WT mice (Fig. 7a \& b), KO mice had significantly fewer adipocytes (Fig. 7a \& c). This 377 suggests that the deletion of Panx3 may reduce the total number of adipocytes in subcutaneous 378 tissue.

379 Considering there is no published literature on PANX3's role in adipose-derived stromal cells 380 (ASCs) or early adipocyte development, we wanted to determine what role Panx3 may be playing 381 in cell proliferation and viability. ASCs were isolated from the inguinal fat pads of WT and KO 382 male mice on a HFD, as described previously (6). ASCs cultured from KO mice grew significantly 383 slower (Fig. 7d) at 4 and 7 days compared to WT ASCs. When ASCs were cultured to induce 384 differentiation to adipocytes, there was a non-significant trend for PANX3 protein expression to 385 increase (Fig. 7e \& f). In a pre-adipocytes cell line (3T3-L1), PANX3 significantly increased 386 during induction to terminal adipocyte differentiation (Fig. $7 \mathrm{~g} \& \mathrm{~h}$ ). These results suggest that

387 Panx3 deletion reduces total fat cell number in adult male mice, reduces ASC growth, and may be 388 involved in adipocyte development as its expression is increased during induction.

\section{Discussion}

390 A number of studies have shown that $\operatorname{Panx} 3$ has a role in the development and pathophysiology

391 of skin $(9,25-28)$, bone $(25,29,30)$, and cartilage $(20,31,32)$, and there have been indirect reports

392 of its involvement in body mass index (9) and adipogenesis (10). In previous publications we 393 examined weight and fat mass differences between WT and Panx3 KO mice at 12 weeks of age 
394 (20) or at later ages (18- and 24- months) (32), and we saw no significant differences between

395 genotypes. In this study we observed large significant differences in weight and fat mass in male

396 KO mice at 24 and 30-weeks of age on a chow diet. We also showed that diet and exercise are

397 regulators of PANX3 expression in mouse adipose tissue, and it is significantly more expressed in

398 male adipose tissue. While there was a genotype effect for female KO mice to weigh less at later

399 time points, the deletion of Panx3 resulted in much larger weight reductions in males. Most of this

400 body weight difference can be accounted for by the lower fat mass in KO mice. This lower fat

401 mass was to the same extent as 6 weeks of FEX in WT mice, however, there were no significant

402 differences in body weight between genotypes when challenged with a HFD. Upon further

403 investigation to determine why Panx3 deletion may reduce fat mass, we found that these mice

404 have a reduced number of adipocytes in their subcutaneous fat. Furthermore, KO mice had lower

405 levels of multiple pro-inflammatory genes in white adipose and skeletal muscle tissue under both

406 regular chow and HFD feeding. These results suggest that PANX3 is expressed at higher levels in

407 male adipose tissue, and may regulate adipocyte cell proliferation, body fat accumulation and

408 inflammatory gene expression in male mice.

409 Differences in obesity rates between males and females is the result of a complex interaction

410 between chromosomal, hormonal, gender and behavioural factors (33). While there were

411 significant differences in body weight between WT and KO females as they aged, deleting Panx3

412 in males had a much more profound effect on body mass and composition. Considering male

413 C57BL/6 mice are much more susceptible to weight gain and fat expansion under a variety of

414 dietary conditions (34), this may explain why we observed a greater effect in males. Furthermore,

415 quantitative trait loci data linking $\operatorname{Panx} 3$ to body mass index were specific to male mice (9) which

416 further supports the findings in this study. Additionally, we found that PANX3 expression was 
417 significantly higher in male adipose tissue compared to females, supporting the notion that Panx3

418 plays a role in male but not female adiposity.

419 Both estrogen and testosterone play a role in metabolic disease and obesity (35). We did not

420 measure sex hormones in this study, and there are no published reports of estrogen and testosterone

421 levels in Panx3 KO mice. However, PANX3 is expressed in Leydig cells (36) and therefore may

422 influence testosterone production. Interestingly, the female Panx3 $\mathrm{KO}$ mice in this study had

423 significantly higher inflammatory indices in quadriceps and liver tissues. This dichotomy in

424 inflammatory changes between male and female KOs is perplexing, however PANX3 may

425 influence inflammation differently between sexes due to gonadal white adipose tissue, which

426 contributes to differences in lipid metabolism and inflammation between sexes (37).

427 We have previously shown, in cultured myotubes, that PANX3 propitiates the cell-intrinsic pro-

428 inflammatory effects of the dietary fatty acid palmitate (11). Blocking of PANX3 channels reduced

429 the capacity of cultured skeletal muscle cells to recruit monocytes. While in the present study we

430 did not quantify immune cells, male Panx3 KO mice had significantly lower expression of Emr1,

431 a macrophage marker, in quadriceps and adipose tissue. Additionally, KO mice had reduced

432 expression of pro-inflammatory relative to anti-inflammatory genes, suggesting that the deletion

433 of Panx3 may attenuate diet and sedentary behaviour induced adipose tissue inflammation (38).

434 While these results support our previous observations regarding PANX3's role in inflammation,

435 we are unable to determine which cell type is responsible for the altered inflammatory expression.

436 Exercise has been shown in both animal and humans to have anti-inflammatory effects

437 systemically and in adipose tissue (39-41). Studies in mouse models show that exercise attenuates

438 visceral white adipose tissue inflammation caused by HFD (42), specifically, the recruitment of 
439 M1-like macrophages and CD8+ T cells upon exposure to HFD (43). In this study, while we did

440 not assess markers of CD8+T cells, we found that FEX in WT mice resulted in significantly lower

441 levels of macrophage markers in skeletal muscle and adipose tissue. Interestingly, the deletion of

442 Panx3 also reduced macrophage markers, and resulted in lowering of multiple pro-inflammatory

443 genes that exercise had no effect on. This would suggest that the deletion of Panx3 has an even

444 greater impact on inflammatory gene expression than 6 weeks of daily FEX.

445 Previously we reported that Panxl KO mice have more fat mass, less lean mass and weigh more

446 than WT mice (6). This suggests an opposite effect than what was observed in the present study

447 with the deletion of Panx3. The potential opposing role of Panxl and Panx3 in adipose tissue is

448 not certain, however this may be due to differing functions of the two pannexin isoforms during

449 early development and their involvement in pre-adipocyte fate. While both ASCs from Panxl and

450 Panx3 KOs have reduced proliferation compared to WT ASCs, Panxl KO ASCs have enhanced

451 adipogenic differentiation. We did not perform any further assays to assess differentiation fate of

452 Panx3 KO ASCs, and future research is necessary to study the function of PANX3 in these cells.

453 Consistent daily exercise is necessary for health, however much of the literature suggests that

454 exercise alone cannot reduce adiposity in people with obesity, and dietary interventions are

455 necessary $(44,45)$. In mouse models, the extent to which exercise can influence body weight may

456 be dependent on the age, sex, diet, and the nature of the exercise intervention (voluntary versus

457 forced) $(46,47)$. We found that FEX attenuated weight gain in WT mice because of reduced fat

458 mass and increased lean mass to body weight ratio. FEX had no additional effect on body weight

459 in Panx3 KO mice however, as these mice do not gain a significant amount of weight or fat mass

460 between 24 and 30 weeks of age. This suggests the presence of the Panx3 gene is necessary for

461 the natural weight gain that occurs in adult male WT mice under sedentary conditions. What is 
462 striking is the magnitude of difference in body weight (difference between means: $7.117 \mathrm{~g} \pm$

4630.6830 ) and fat mass (difference between means: $4.727 \mathrm{~g} \pm 1.238$ ) between genotypes. This equates

464 to an approximately $46.8 \%$ reduction in fat mass, which is like the effect of FEX in this experiment.

465 While we saw drastic effects on body and fat mass from the deletion of Panx3 in males under SED

466 and regular chow fed conditions, there were no significant differences in body weight during HFD

467 feeding. This finding is in line with multiple previous reports that obesity is mainly the result of

468 excess caloric intake $(48,49)$. However, we know individuals can vary in how much weight they

469 gain while in a similar caloric excess (50) which would suggest genetic and behavioural factors

470 are also at play. Our findings highlight the importance of taking into consideration environmental

471 and behavioural factors that can interact with genetics when investigating multifactorial diseases

472 such as obesity. Manipulating Panx3 may not be effective when consuming an excessive caloric

473 diet, however it may be an effective target for patients who are also engaging in healthy caloric

474 consumption.

475 While Panx3 levels were low in adipose tissue of chow fed WT animals, it was significantly

476 elevated in mice fed a Western or HFD. This suggests that Panx3 expression is sensitive to

477 dietary factors, as gleaned from previous work in cell culture models (11). Moreover, FEX was

478 able to counter this diet induced Panx3 upregulation. This would suggest that exercise is able to

479 inhibit the signalling responsible for PANX3 expression caused by dietary factors. Future studies

480 will be needed to determine what signaling pathways are responsible for the induction and

481 suppression of PANX3 expression by diet and exercise. However, our previous data along with

482 those reported by others indicate that the toll-like receptor 4 (TLR4)/nuclear factor $-\kappa B(N F-\kappa B)$

483 pathway is activated by the saturated fatty acid palmitate (51). We previously showed that this

484 pathway mediated the expression of Panx3 mRNA (11). Conversely, moderate aerobic exercise 
485 is known to downregulate TLR4, and consequently the proinflammatory NF- $\kappa \mathrm{B}$ pathway, thus,

486 potentially inhibiting Panx3 expression (52).

\section{Conclusion}

488 We have shown that the deletion of Panx3 attenuates body weight gain because of lower fat mass

489 in male mice. Additionally, skeletal muscle and adipose tissue of KO mice shift to a more anti-

490 inflammatory phenotype in males. This effect was equivalent to the reduction in body weight gain

491 and fat mass reduction caused by 6 weeks of daily FEX. This suggests PANX3 plays a significant

492 role in fat accumulation and inflammation in adult male mice. This phenotype may be the result

493 of PANX3's role in adipocyte proliferation in early life. Considering this study used a global KO

494 model, future research is needed to determine if PANX3 functions in other cell types involved in

495 this phenotype. Manipulating PANX3 channel function or expression may be a potential

496 therapeutic target in conjunction with dietary and exercise interventions to manage obesity and

497 associated inflammation in males.

\section{Author Contributions}

499 CBW project design, mouse husbandry, research data, data analysis, wrote manuscript; VRL

500 research data, data analysis, edited manuscript; DJ research data, reviewed manuscript; PB

501 research data, data analysis; NJP research data, data analysis, edited manuscript; SS, BOD, JT,

502 RESP research data, data analysis, edited manuscript; KJB research data, mouse husbandry,

503 reviewed and edited manuscript; RG research data, metabolic cage analysis; LF research data,

504 edited manuscript; NB research data, edited manuscript; AK research data analysis, manuscript

505 review and editing; FB project design, manuscript review and editing, funding; SP project design

506 and supervision, data analysis, funding, manuscript review and editing. 


\section{Acknowledgements and conflict of interest statement}

508 The authors declare no conflict of interest of any kind with the current manuscript. We thank the

509 funding agencies that supported this work including: Petro-Canada Young Innovator Award -

510 Western University to SP, Ontario Graduate Scholarship to CBW. F.B holds the Canada Research

511 Chair in Musculoskeletal Research and is the recipient of a Foundation Grant from the Canadian

512 Institutes of Health Research (CIHR, Grant \#332438). CIHR Foundation Grant (FRN:FDN-

513143203 ) to AK. Dr. Silvia Penuela is the guarantor of this work and, as such, had full access to all

514 the data in the study and takes responsibility for the integrity of the data and the accuracy of the

515 data analysis.

\section{Data availability}

517 All data generated or analyzed during this study are included in the published article (and its online

518 supplementary files). Raw data is available from the corresponding author upon reasonable 519 request. 


\section{References}

1. Romieu I, Dossus L, Barquera S, Blottière HM, Franks PW, Gunter M, et al. Energy balance and obesity: what are the main drivers? Cancer Causes Control. 2017;28(3):247-58. 2. Hall KD, Kahan S. Maintenance of lost weight and long-term management of obesity. Med Clin North Am. 2018;102(1):183-97.

3. Thaker VV. Genetic and epigenetics causes of obesity. Adolesc Med State Art Rev. 2017;28(2):379-405.

4. Penuela S, Gehi R, Laird DW. The biochemistry and function of pannexin channels. Biochim Biophys Acta. 2013;1828(1):15-22.

5. Tozzi M, Hansen JB, Novak I. Pannexin-1 mediated ATP release in adipocytes is sensitive to glucose and insulin and modulates lipolysis and macrophage migration. Acta Physiol (Oxf). 2020;228(2):e13360.

6. Lee VR, Barr KJ, Kelly JJ, Johnston D, Brown CFC, Robb KP, et al. Pannexin 1 regulates adipose stromal cell differentiation and fat accumulation. Sci Rep. 2018;8(1):16166. 7. Adamson SE, Meher AK, Chiu YH, Sandilos JK, Oberholtzer NP, Walker NN, et al. Pannexin 1 is required for full activation of insulin-stimulated glucose uptake in adipocytes. Mol Metab. 2015;4(9):610-8.

8. Senthivinayagam S, Serbulea V, Upchurch CM, Polanowska-Grabowska R, Mendu SK, Sahu S, et al. Adaptive thermogenesis in brown adipose tissue involves activation of pannexin-1 channels. Mol Metab. 2020;44:101130.

9. Halliwill KD, Quigley DA, Kang HC, Del Rosario R, Ginzinger D, Balmain A. Panx3 links body mass index and tumorigenesis in a genetically heterogeneous mouse model of carcinogen-induced cancer. Genome Med. 2016;8(1):83.

10. Sjölund J, Pelorosso FG, Quigley DA, DelRosario R, Balmain A. Identification of Hipk2 as an essential regulator of white fat development. Proc Natl Acad Sci U S A. 2014;111(20):7373-8.

11. Pillon NJ, Li YE, Fink LN, Brozinick JT, Nikolayev A, Kuo MS, et al. Nucleotides released from palmitate-challenged muscle cells through pannexin-3 attract monocytes. Diabetes. 2014;63(11):3815-26.

12. Goossens GH. The metabolic phenotype in obesity: fat mass, body fat distribution, and adipose tissue function. Obes Facts. 2017;10(3):207-15.

13. Reilly SM, Saltiel AR. Adapting to obesity with adipose tissue inflammation. Nat Rev Endocrinol. 2017;13(11):633-43.

14. Stolarczyk E. Adipose tissue inflammation in obesity: a metabolic or immune response? Curr Opin Pharmacol. 2017;37:35-40.

15. Unamuno X, Gómez-Ambrosi J, Rodríguez A, Becerril S, Frühbeck G, Catalán V. Adipokine dysregulation and adipose tissue inflammation in human obesity. Eur J Clin Invest. 2018;48(9):e12997.

16. Burhans MS, Hagman DK, Kuzma JN, Schmidt KA, Kratz M. Contribution of adipose tissue inflammation to the development of type 2 diabetes mellitus. Compr Physiol. 2018;9(1):158 .

17. Hotamisligil GS. Inflammation and metabolic disorders. Nature. 2006;444(7121):860-7. 18. Kusminski CM, Bickel PE, Scherer PE. Targeting adipose tissue in the treatment of obesity-associated diabetes. Nat Rev Drug Discov. 2016;15(9):639-60. 

obesity, diabetes, and blood pressure: role of perivascular adipose tissue. Physiol Rev. 2019;99(4):1701-63.

20. Moon PM, Penuela S, Barr K, Khan S, Pin CL, Welch I, et al. Deletion of Panx3 prevents the development of surgically induced osteoarthritis. J Mol Med (Berl). 2015;93(8):845-56. of pannexins on noise-induced hearing loss. Biochem J. 2016;473(24):4665-80. 22. Penuela S, Bhalla R, Gong XQ, Cowan KN, Celetti SJ, Cowan BJ, et al. Pannexin 1 and pannexin 3 are glycoproteins that exhibit many distinct characteristics from the connexin family of gap junction proteins. J Cell Sci. 2007;120(Pt 21):3772-83.

23. Yu G, Wu X, Kilroy G, Halvorsen YD, Gimble JM, Floyd ZE. Isolation of murine adipose-derived stem cells. Methods Mol Biol. 2011;702:29-36.

24. Gao M, Ma Y, Liu D. High-fat diet-induced adiposity, adipose inflammation, hepatic steatosis and hyperinsulinemia in outbred CD-1 mice. PLoS One. 2015;10(3):e0119784.

25. Abitbol JM, O'Donnell BL, Wakefield CB, Jewlal E, Kelly JJ, Barr K, et al. Double deletion of Panx1 and Panx3 affects skin and bone but not hearing. J Mol Med (Berl). 2019;97(5):723-36.

26. Celetti SJ, Cowan KN, Penuela S, Shao Q, Churko J, Laird DW. Implications of pannexin 1 and pannexin 3 for keratinocyte differentiation. J Cell Sci. 2010;123(Pt 8):1363-72. 27. Zhang P, Ishikawa M, Doyle A, Nakamura T, He B, Yamada Y. Pannexin 3 regulates skin development via Epiprofin. Sci Rep. 2021;11(1):1779.

28. Zhang P, Ishikawa M, Rhodes C, Doyle A, Ikeuchi T, Nakamura K, et al. Pannexin-3 deficiency delays skin wound healing in mice due to defects in channel functionality. J Invest Dermatol. 2019;139(4):909-18.

29. Bond SR, Lau A, Penuela S, Sampaio AV, Underhill TM, Laird DW, et al. Pannexin 3 is a novel target for Runx2, expressed by osteoblasts and mature growth plate chondrocytes. J Bone Miner Res. 2011;26(12):2911-22.

30. Ishikawa M, Williams G, Forcinito P, Ishikawa M, Petrie RJ, Saito K, et al. Pannexin 3 $\mathrm{ER} \mathrm{Ca}(2+)$ channel gating is regulated by phosphorylation at the Serine 68 residue in osteoblast differentiation. Sci Rep. 2019;9(1):18759.

31. Iwamoto T, Nakamura T, Doyle A, Ishikawa M, de Vega S, Fukumoto S, et al. Pannexin 3 regulates intracellular ATP/cAMP levels and promotes chondrocyte differentiation. J Biol Chem. 2010;285(24):18948-58.

32. Moon PM, Shao ZY, Wambiekele G, Appleton C, Laird DW, Penuela S, et al. Global deletion of pannexin 3 accelerates development of aging-induced osteoarthritis in mice. Arthritis Rheumatol. 2021.

601

602

603

604

605

606

607

608

609 33. Link JC, Reue K. Genetic basis for sex differences in obesity and lipid metabolism. Annu Rev Nutr. 2017;37:225-45.

34. Ingvorsen C, Karp NA, Lelliott CJ. The role of sex and body weight on the metabolic effects of high-fat diet in C57BL/6N mice. Nutr Diabetes. 2017;7(4):e261.

35. Shin JH, Hur JY, Seo HS, Jeong YA, Lee JK, Oh MJ, et al. The ratio of estrogen receptor alpha to estrogen receptor beta in adipose tissue is associated with leptin production and obesity. Steroids. 2007;72(6-7):592-9.

610 36. Turmel P, Dufresne J, Hermo L, Smith CE, Penuela S, Laird DW, et al. Characterization of pannexin1 and pannexin 3 and their regulation by androgens in the male reproductive tract of the adult rat. Mol Reprod Dev. 2011;78(2):124-38. 
611 37. Varghese M, Griffin C, McKernan K, Eter L, Lanzetta N, Agarwal D, et al. Sex

612 differences in inflammatory responses to adipose tissue lipolysis in diet-induced obesity.

613 Endocrinology. 2019;160(2):293-312.

614 38. Winn NC, Cottam MA, Wasserman DH, Hasty AH. Exercise and adipose tissue

615 immunity: outrunning inflammation. Obesity. 2021;29(5):790-801.

616 39. Paolucci EM, Loukov D, Bowdish DME, Heisz JJ. Exercise reduces depression and

617 inflammation but intensity matters. Biol Psychol. 2018;133:79-84.

618 40. Petersen AM, Pedersen BK. The anti-inflammatory effect of exercise. J Appl Physiol

619 (1985). 2005;98(4):1154-62.

620 41. Suzuki K. Chronic inflammation as an immunological abnormality and effectiveness of

621 Exercise. Biomolecules. 2019;9(6).

622 42. Rosa-Neto JC, Silveira LS. Endurance exercise mitigates immunometabolic adipose

623 tissue disturbances in cancer and obesity. Int J Mol Sci. 2020;21(24).

624 43. Kawanishi N, Yano H, Yokogawa Y, Suzuki K. Exercise training inhibits inflammation

625 in adipose tissue via both suppression of macrophage infiltration and acceleration of phenotypic

626 switching from M1 to M2 macrophages in high-fat-diet-induced obese mice. Exerc Immunol

627 Rev. 2010;16:105-18.

628 44. Swift DL, Johannsen NM, Lavie CJ, Earnest CP, Church TS. The role of exercise and

629 physical activity in weight loss and maintenance. Prog Cardiovasc Dis. 2014;56(4):441-7.

630 45. Swift DL, McGee JE, Earnest CP, Carlisle E, Nygard M, Johannsen NM. The effects of

631 exercise and physical activity on weight loss and maintenance. Prog Cardiovasc Dis.

632 2018;61(2):206-13.

633 46. Carpenter KC, Strohacker K, Breslin WL, Lowder TW, Agha NH, McFarlin BK. Effects

634 of exercise on weight loss and monocytes in obese mice. Comp Med. 2012;62(1):21-6.

635 47. McCabe LR, Irwin R, Tekalur A, Evans C, Schepper JD, Parameswaran N, et al. Exercise

636 prevents high fat diet-induced bone loss, marrow adiposity and dysbiosis in male mice. Bone.

637 2019;118:20-31.

638 48. Golay A, Bobbioni E. The role of dietary fat in obesity. Int J Obes Relat Metab Disord.

639 1997;21 Suppl 3:S2-11.

640 49. Wang L, Wang H, Zhang B, Popkin BM, Du S. Elevated fat intake increases body weight

641 and the risk of overweight and obesity among chinese adults: 1991-2015 Trends. Nutrients.

642 2020;12(11).

643 50. Levine JA, Eberhardt NL, Jensen MD. Role of nonexercise activity thermogenesis in

644 resistance to fat gain in humans. Science. 1999;283(5399):212-4.

645 51. Ajuwon KM, Spurlock ME. Palmitate activates the NF-kappaB transcription factor and

646 induces IL-6 and TNFalpha expression in 3T3-L1 adipocytes. J Nutr. 2005;135(8):1841-6.

647 52. Cavalcante PAM, Gregnani MF, Henrique JS, Ornellas FH, Araújo RC. Aerobic but not

648 resistance exercise can induce inflammatory pathways via toll-like 2 and 4: a systematic review.

649 Sports Med Open. 2017;3(1):42. 
Figure 1: Male Panx3 KO mice weigh less, have less fat mass and more lean mass than WT mice to the same extent as 6 weeks of forced exercise.

655 Visual graphic of the experimental design. Male and female WT and Panx3 KO (KO) mice were 656 randomly allocated to either SED or FEX groups from 24 (baseline) to 30 weeks of age (6 weeks) (a). Body weights were measured biweekly, and blood glucose and body composition were measured at baseline and 30 weeks of age. After which, blood and metabolic organs were collected for ex vivo analysis. Male body weight development from 4 to 30 weeks of age (b). Baseline comparisons between (checkered bars) KO mice (red, $\mathrm{n}=15$ ) and WT mice (blue, $\mathrm{n}=19$ ), and at 30 weeks of age for SED (clear bars) and FEX (solid bars) for body weight (c), fat mass (d), fat mass corrected for body weight (e), lean mass (f), and lean mass corrected for body weight (g). Results are expressed as mean \pm SEM. A three-way ANOVA was conducted with activity $x$ genotype $\mathrm{x}$ age as factors to determine significant differences between the genotypes for each group $(\mathrm{n}=3-5)$. Different letters indicate significantly different groups $(\mathrm{p}<0.05)$. ns: nonsignificant. WT SED: wildtype sedentary, KO SED: Panx3 knockout sedentary, WT FEX: wildtype forced exercise, KO FEX: Panx3 knockout forced exercise.

668 Figure 2: Male Panx3 KO mice have lower inflammatory index in quadriceps and visceral 669 fat tissues compared to WT mice.

670 WT (blue bars) and KO (red bars) mice were allocated to either SED (clear bars) or FEX (solid bars) groups from 24 (baseline) to 30 weeks of age. Quadriceps (a \& b), visceral fat (c \& d), and liver (e \& f) tissues were collected, and mRNA expression was analyzed by RT-qPCR for macrophage markers Emrl and Itgax (CD11c), pro-inflammatory genes Tnfo, Nos2, Il12a, Ccl2 and $\mathrm{IlO}$ and anti-inflammatory markers $\mathrm{Arg} 1, \mathrm{Mrcl}, \mathrm{IllO}$ and Chi3l3. An inflammatory index score was calculated as the ratio of the sum of the pro-inflammatory over the sum of the antiinflammatory markers and reflects the inflammatory status of the tissue (b, d, f). A two-way ANOVA was conducted with genotype $\mathrm{x}$ activity as factors. $\mathrm{n}=3-5$. mean \pm SEM. Different letters indicate significantly different means $(\mathrm{p}<0.05)$. ns: non-significant. a.u.: arbitrary units. WT SED: wildtype sedentary, KO SED: Panx3 knockout sedentary, WT FEX: wildtype forced exercise, KO

680 FEX: Panx3 knockout forced exercise.

681 Figure 3: Female Panx3 KO mice weigh less than WT mice with no significant differences in 682 body fat or lean mass.

683 Female WT (blue) and KO (red) mice were randomly allocated to either SED (clear bars) or FEX (solid bars) group from 24 (baseline) to 30 weeks of age (6 weeks). Body weights were measured biweekly and body composition was measured at baseline and 30 weeks of age. Female body weight measurements from 4-30 weeks age (a). Female body weight comparison at baseline (checkered bars) and 30 weeks of age $(\mathrm{N}=7-13)$ (b). Fat mass (c), fat mass normalized to body weight (d), lean mass (e), and lean mass normalized to body weight (f) was determined by echoMRI. Results are expressed as mean \pm SEM. An unpaired t-test was conducted to assess significant differences between genotypes at baseline of age $(\mathrm{N}=7-8)$. A three-way ANOVA was conducted with activity $\mathrm{x}$ genotype $\mathrm{x}$ age as factors to determine significant differences between the genotypes $(\mathrm{n}=3-4)$. WT SED: wildtype sedentary, KO SED: Panx3 knockout sedentary, WT 
695 Figure 4: Female Panx3 KO mice have higher inflammatory index in quadriceps and liver 696 tissues compared to WT mice.

697 WT (blue) and KO (red) mice were allocated to either SED (clear bars) or FEX (solid bars) groups 698 from 24 to 30 weeks of age. Quadriceps ( $\&$ b), visceral fat (c \& d), and liver (e \& f) tissues were collected, and mRNA expression was analyzed by RT-qPCR for macrophage markers Emrl and Itgax (CD11c), pro-inflammatory genes Tnfo, Nos2, Il12a, Ccl2 and Il6 and anti-inflammatory markers Arg 1, Mrcl, IllO and Chi3l3. An inflammatory index score was calculated as the ratio of the sum of pro-inflammatory over the sum of anti-inflammatory markers and reflects the inflammatory status of the tissue (b, $d, f)$. A two-way ANOVA with genotype $\mathrm{x}$ activity as factors was conducted. $\mathrm{n}=3-5$. ns: not significant, mean \pm SEM. arbitrary units (a.u.). Different letters indicate significantly different means $(\mathrm{p}<0.05)$. WT SED: wildtype sedentary, KO SED: Panx3 knockout sedentary, WT FEX: wildtype forced exercise, KO FEX: Panx3 knockout forced exercise.

Figure 5: PANX3 expression is higher in male visceral fat tissue compared to females, and is regulated by FEX and dietary fat intake.

Male and female WT mice were fed a normal chow diet and allocated to either SED or FEX groups, and their visceral fat was isolated and analysed for PANX3 protein expression ( $\mathrm{a}$ \& b). Protein from animals fed a Western diet $(45 \% \mathrm{kcal}$ from fat) was used as a positive control. mRNA from visceral fat of male mice fed a chow or Western diet and subjected to either the SED or FEX protocol was analysed for Panx3 expression (c \& d). Male WT and Panx3 KO (KO) mice were fed a high fat diet (HFD, $60 \%$ kcal from fat) from 12 to 28 weeks of age and epididymal fat was analysed for PANX3 protein expression (e \& f). KO mouse tissues were used as a negative control (e \& f). ns: not significant, $\mathrm{N}=3, \mathrm{n}=3$. Different letters indicate significantly different means ( $\mathrm{p}$ $<0.05)$. GAPDH was used as a loading control for Western blots, while $18 s$ and Hprt was used for housekeeping genes for qPCR. Body weight $(\mathrm{g})$ and body weight $\%$ change $(\mathrm{h})$ was measured in male mice to determine differences in weight gain between genotypes on a HFD. A two-way repeated measures ANOVA with genotype $\mathrm{x}$ age was conducted $(\mathrm{N}=13-16)$. Results are expressed as mean \pm SEM.

Figure 6: Male Panx3 KO mice are protected from HFD induced inflammation compared to 724 WT mice.

WT and Panx3 KO (KO) mice were fed a HFD (60\% kcal from fat) from 12 to 28 weeks of age. Quadriceps (a \& b), epididymal white fat (eWAT) (c \& d), and liver (e \& f) tissues were collected, and mRNA expression was analyzed by RT-qPCR for macrophage markers Emrl and Itgax (CD11c), pro-inflammatory genes $\operatorname{Tnf} \alpha, \mathrm{Nos} 2, \mathrm{Il} 12 \mathrm{a}, \mathrm{Ccl} 2$ and $\mathrm{Il} 6$ and anti-inflammatory markers Arg 1, Mrc1, IllO and Chi3l3. An inflammatory index score was calculated as the ratio of the sum of pro-inflammatory over the sum of the anti-inflammatory markers and reflects the inflammatory status of the tissue. An unpaired t-test was conducted to determine significant differences between genotypes. $\mathrm{N}=5, *=\mathrm{p}<0.05$. Results are expressed as mean \pm SEM. ns: non-significant, arbitrary units (a.u.).

734 Figure 7: Panx3 KO mice have fewer adipocytes and their primary adipose stromal cells 735 (ASCs) grow slower than those isolated from WT mice. 
736 Representative images of the subcutaneous fat of male WT and Panx3 KO mice (Scale bar $=100$ $737 \mu \mathrm{m})(\mathrm{a})$. Adipocyte size (normalized to WT size) (b) and the number of cells (normalized to the 738 standardized area of view) (c) were quantified. ASCs were isolated from WT and Panx3 KO

739 mice and placed in growth media (d). Western blot and quantification showing PANX3 protein 740 expression in ASCs under controlled and induced conditions (for adipocyte differentiation) (e \& 741 f). PANX3 expression in terminal differentiated 3T3-L1 pre-adipocytes as shown by Western 742 blots of 3T3-L1 cells cultured under controlled and induced conditions ( $\mathrm{g}$ ) and the quantification 743 of PANX3 protein expression (h). $\mathrm{N}=3, \mathrm{n}=3, \mathrm{p}<0.05$. Results are expressed as mean $\pm \mathrm{SEM}$. $744 * \mathrm{p}<0.05, * * * * \mathrm{p}<0.0001$. ns: non-significant. Overexpressing HEK293 cells were used as 745 positive controls (+). 
Figure 1

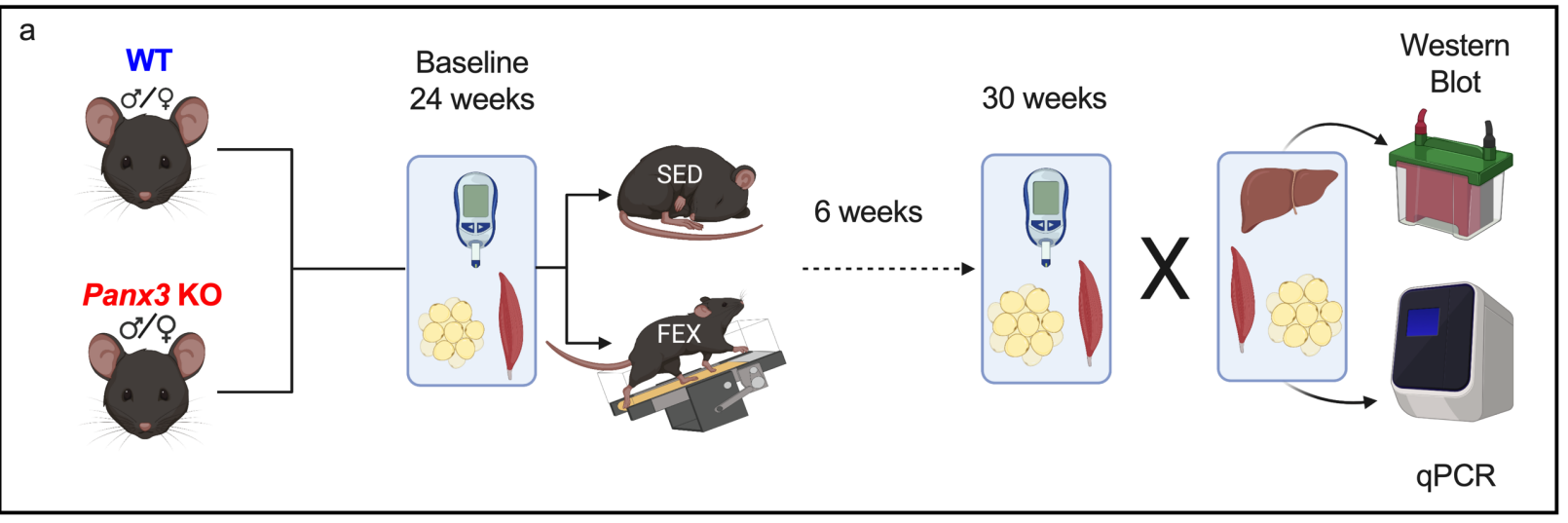

Males

b

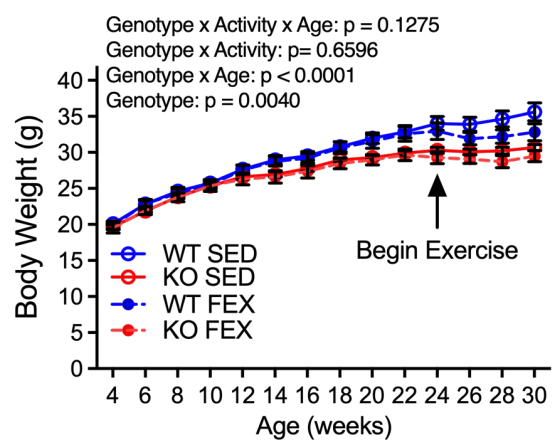

e

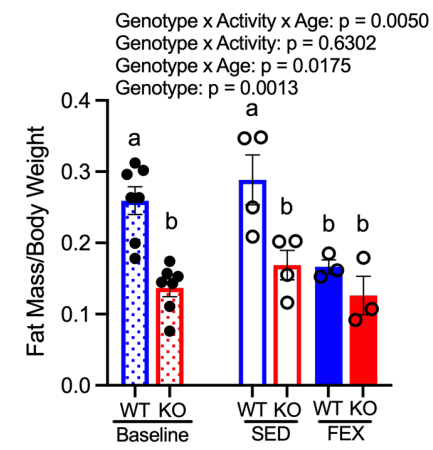

C

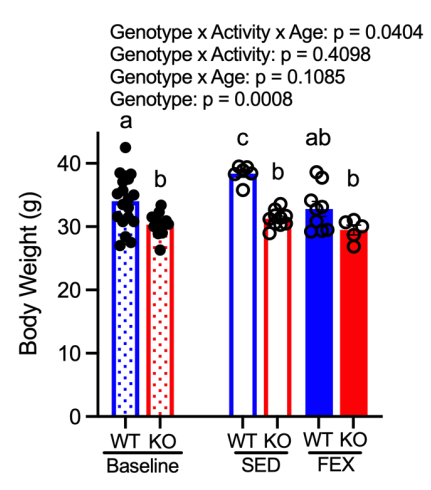

f

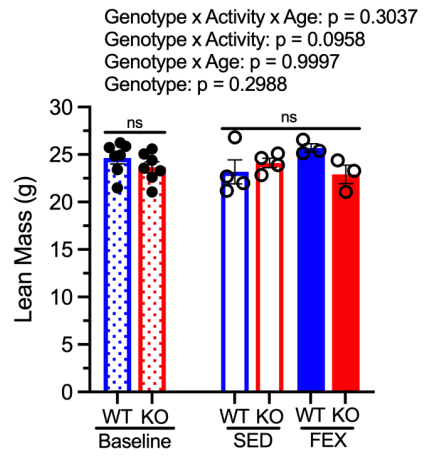

d

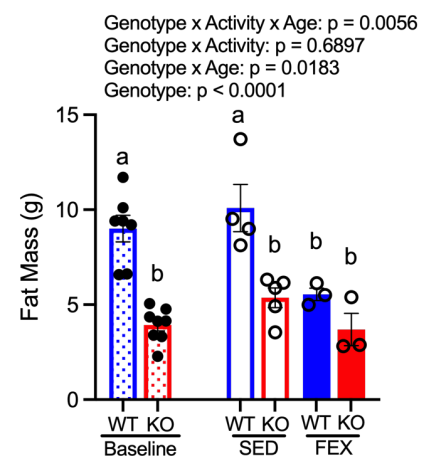

g

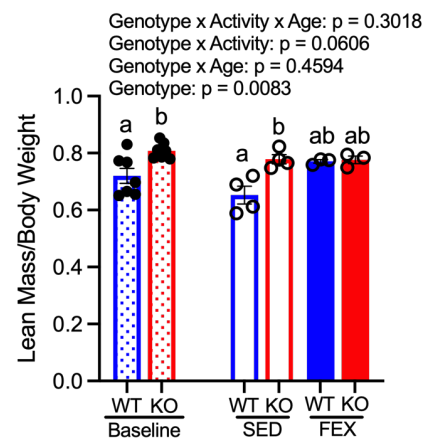




\section{Figure 2}

a

b

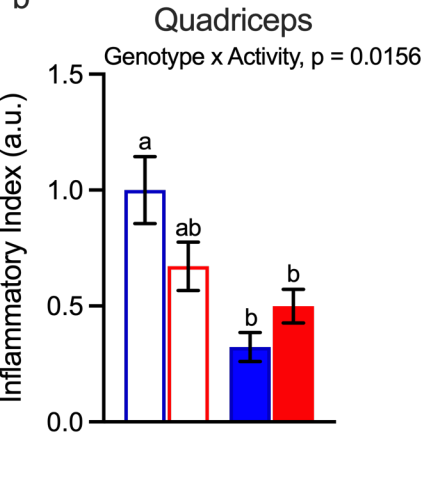

C

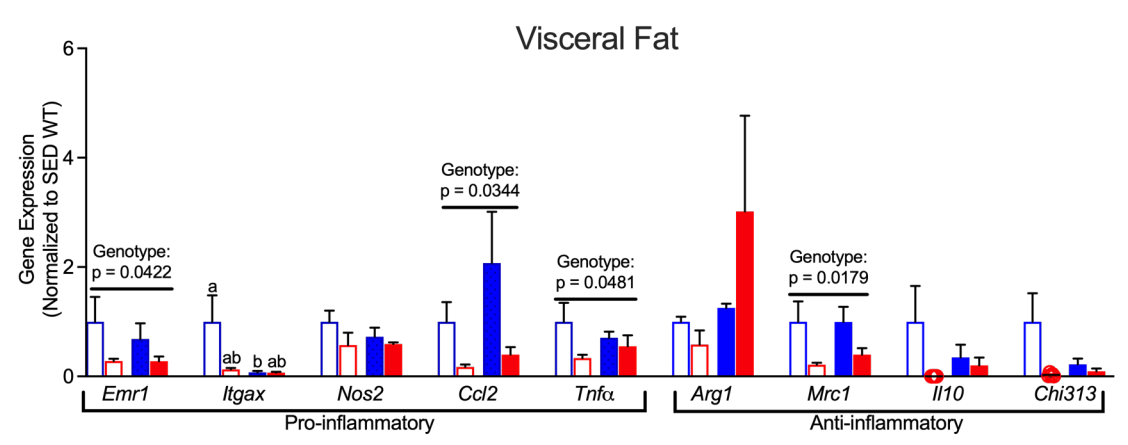

e

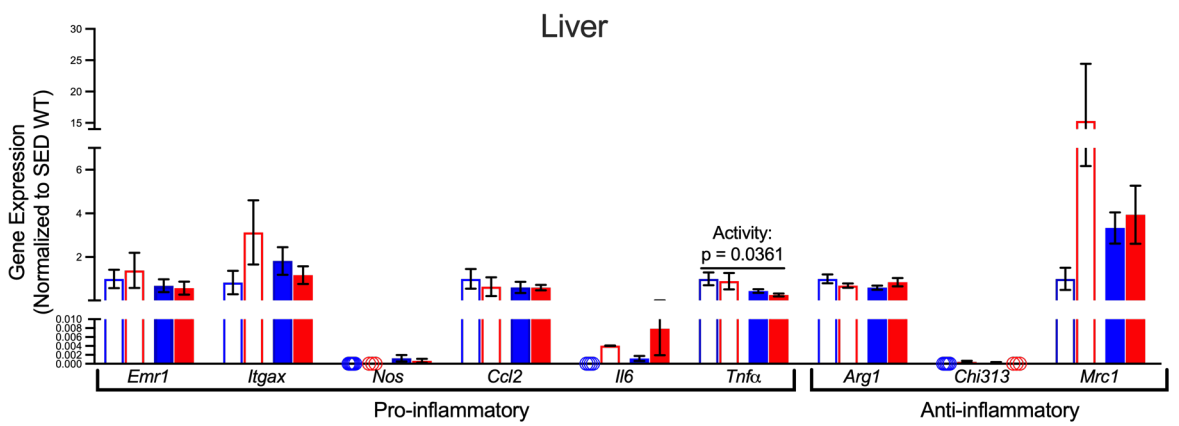

d Visceral Fat

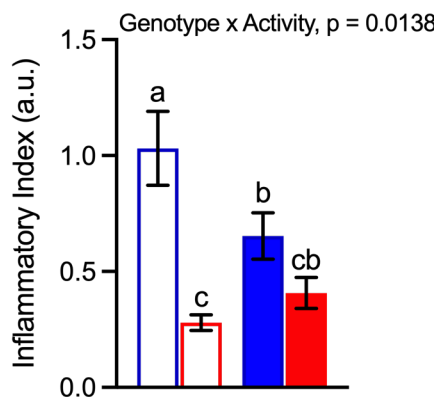

Liver

f Genotype $x$ Activity: $p=0.6094$ Genotype: $p=0.7741$

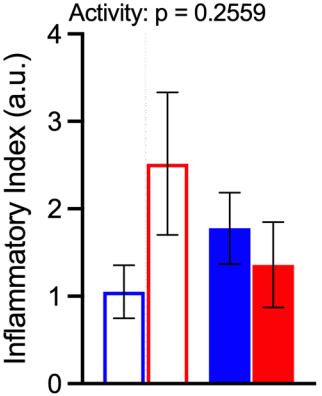


Figure 3

\section{Females}

a

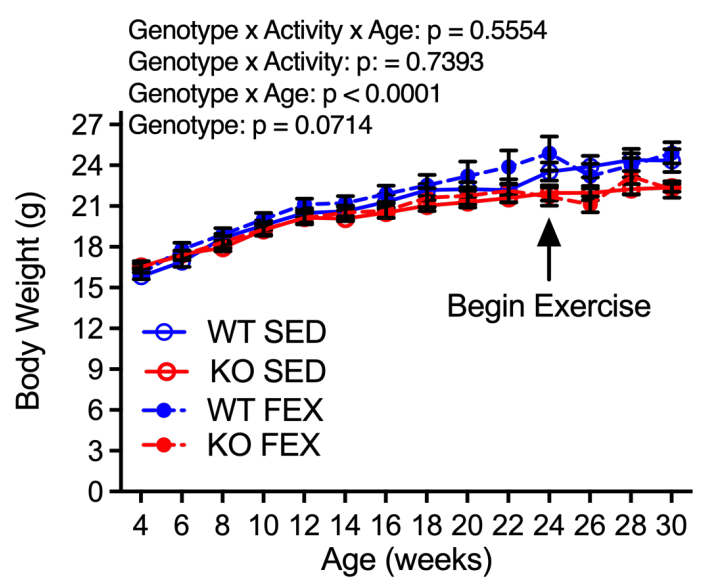

C

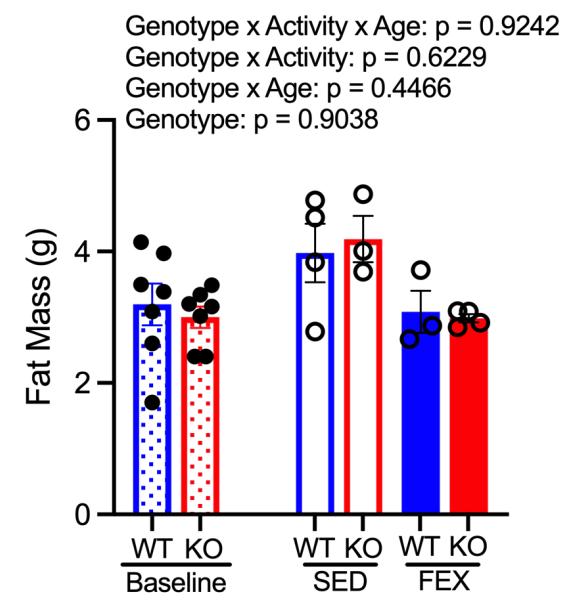

e Genotype $\times$ Activity $\times$ Age: $p=0.3843$

Genotype $x$ Activity: $p=0.7742$

Genotype $x$ Age: $p=0.6661$

Genotype: $p=0.7656$

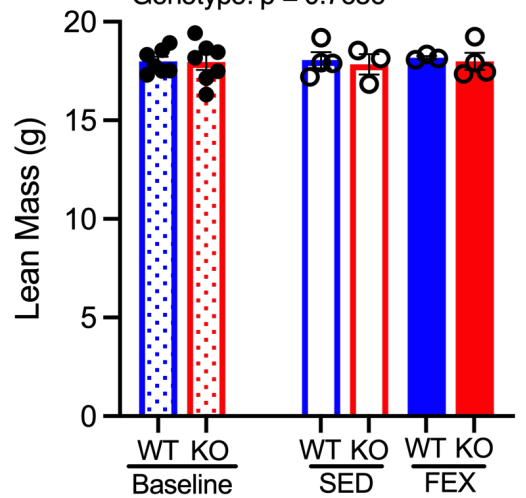

b

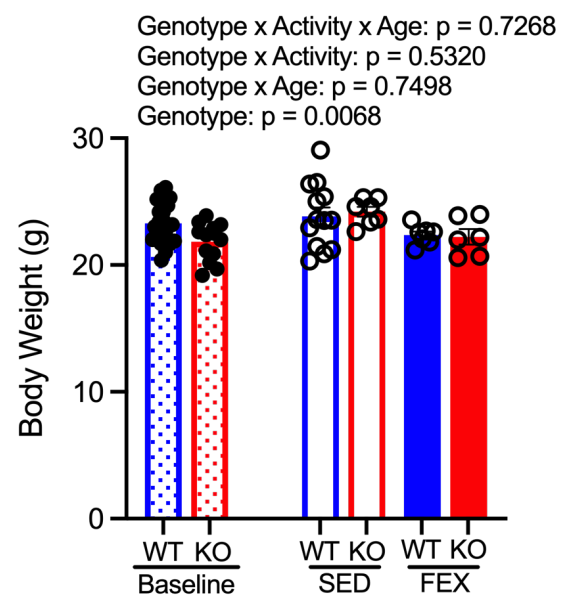

d

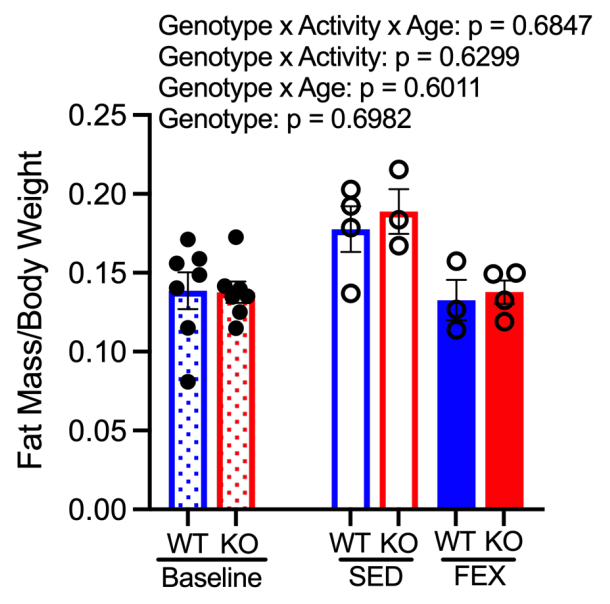

f

Genotype x Activity x Age: $p=0.2294$

Genotype $x$ Activity: $p=0.5047$

Genotype $x$ Age: $p=0.4880$

Genotype: $p=0.2225$

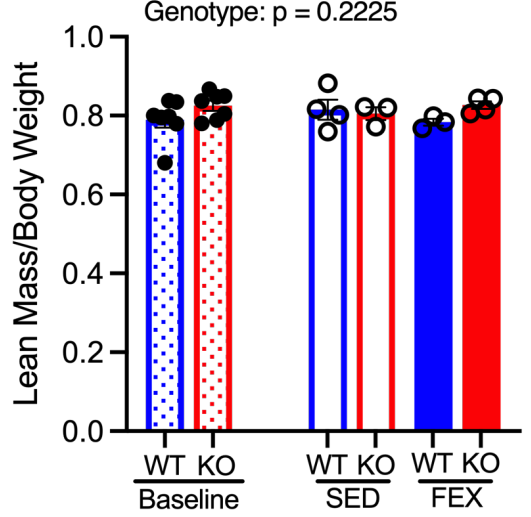


Figure 4

$\begin{array}{lc} & \square \text { WT SED } \\ \text { Females } & \square \text { KO SED } \\ \text { WT FEX } \\ \text { KO FEX }\end{array}$

a

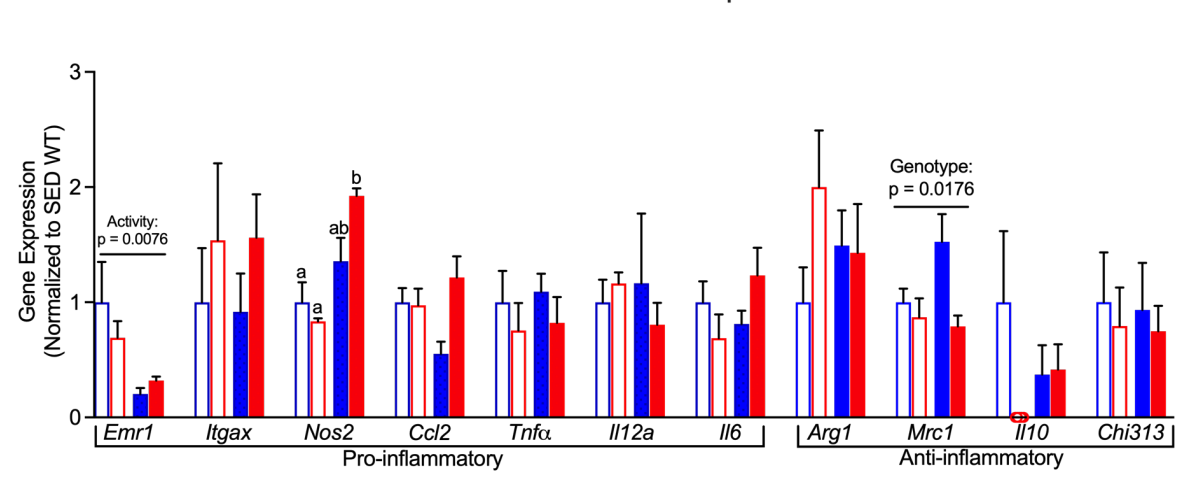

b

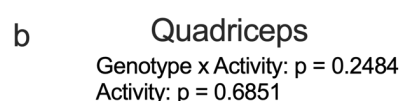

Activity: $p=0.6851$

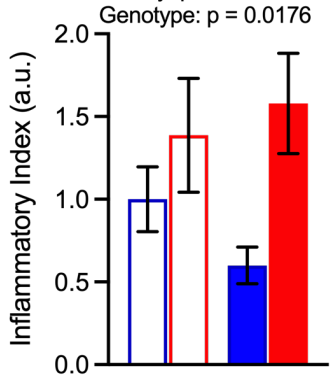

C

Visceral Fat

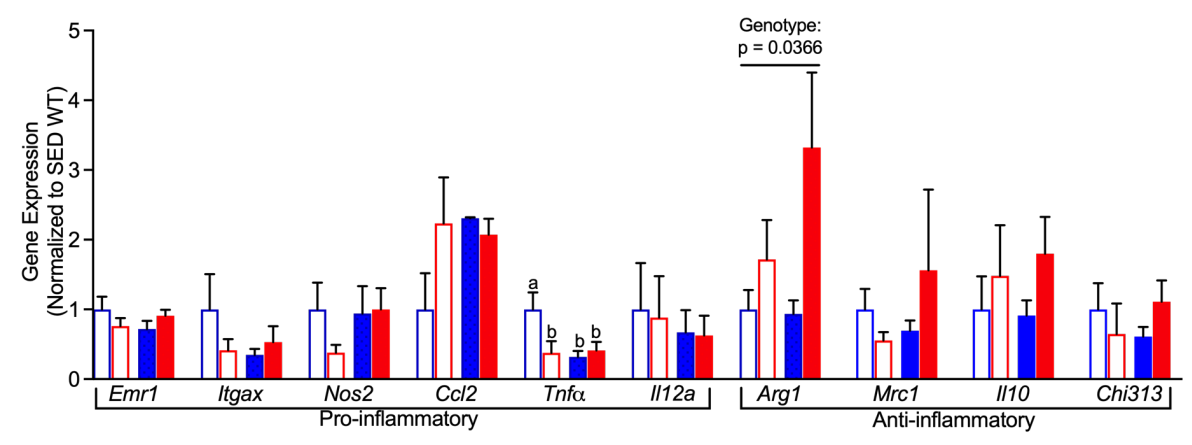

e

Liver

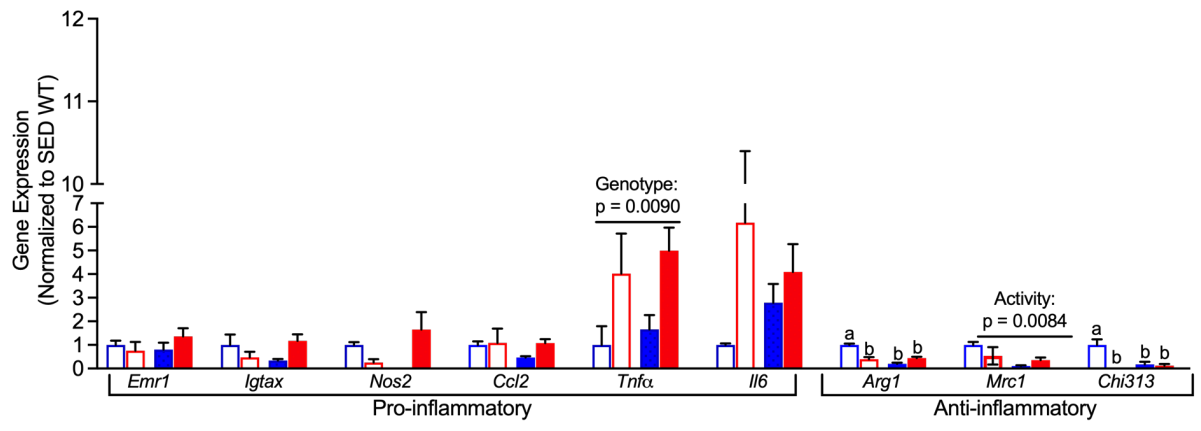

d Visceral Fat Genotype $x$ Activity: $p=0.1017$ Activity: $p=0.4237$

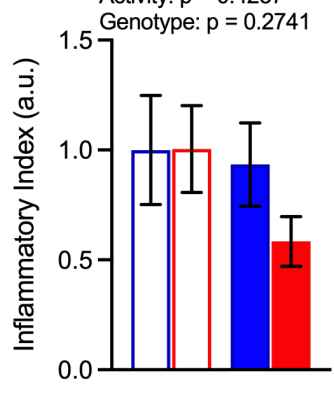

f

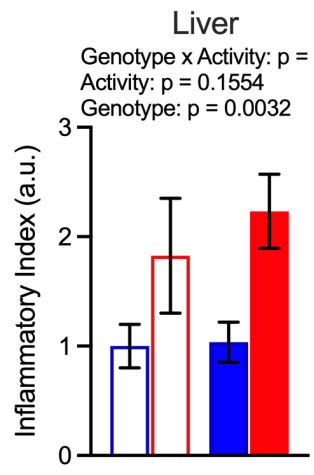




\section{Figure 5}

a

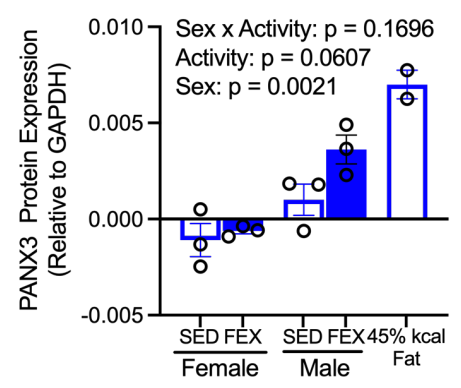

C

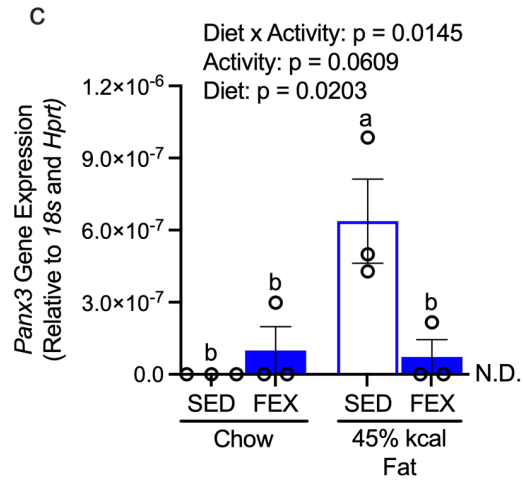

e

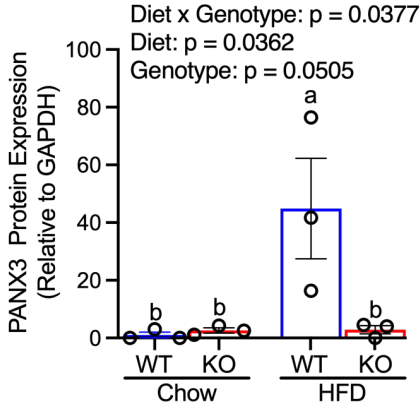

b

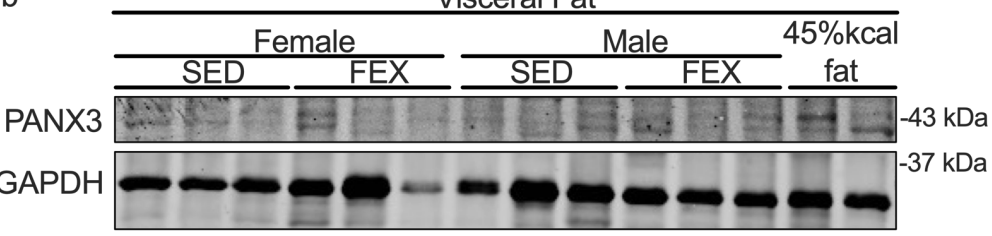

d

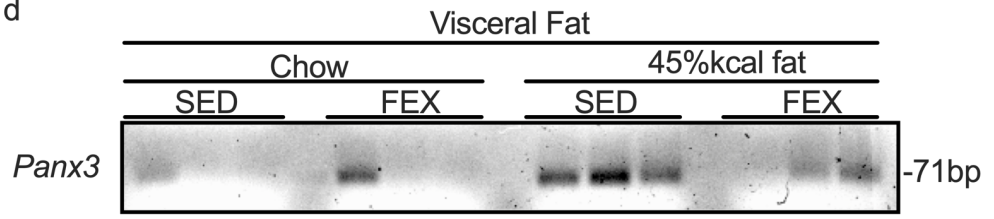

g

h
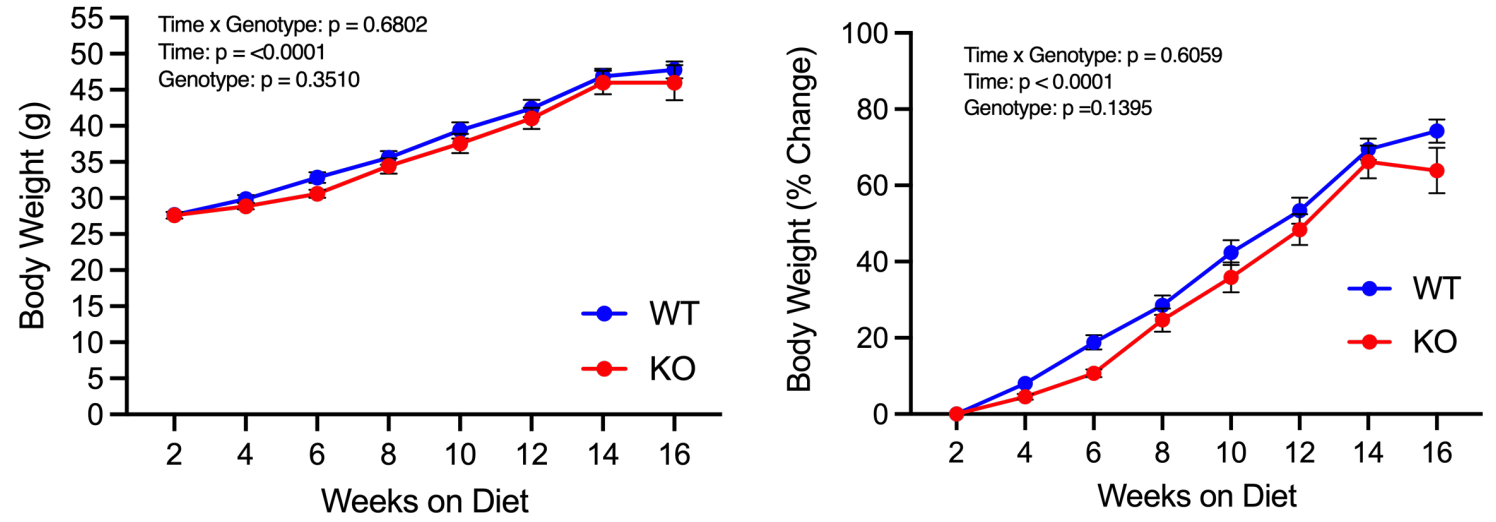
Figure 6

a

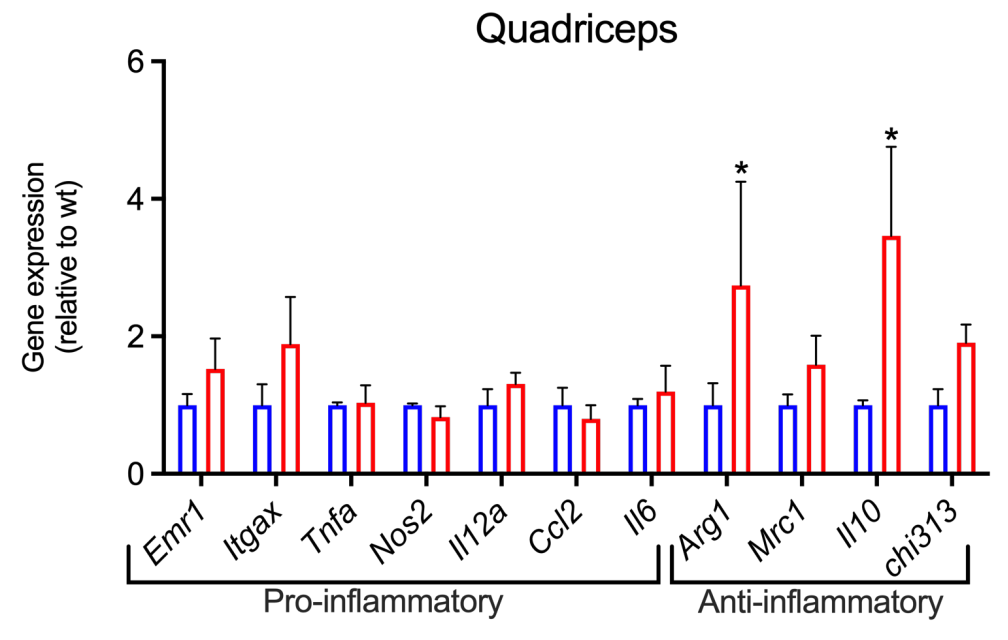

C

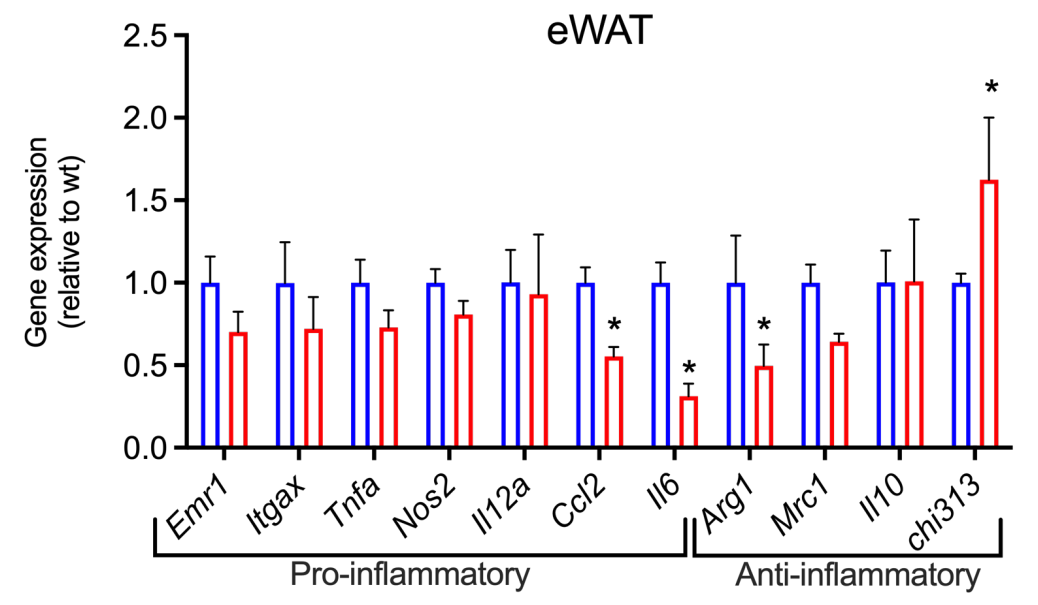

e

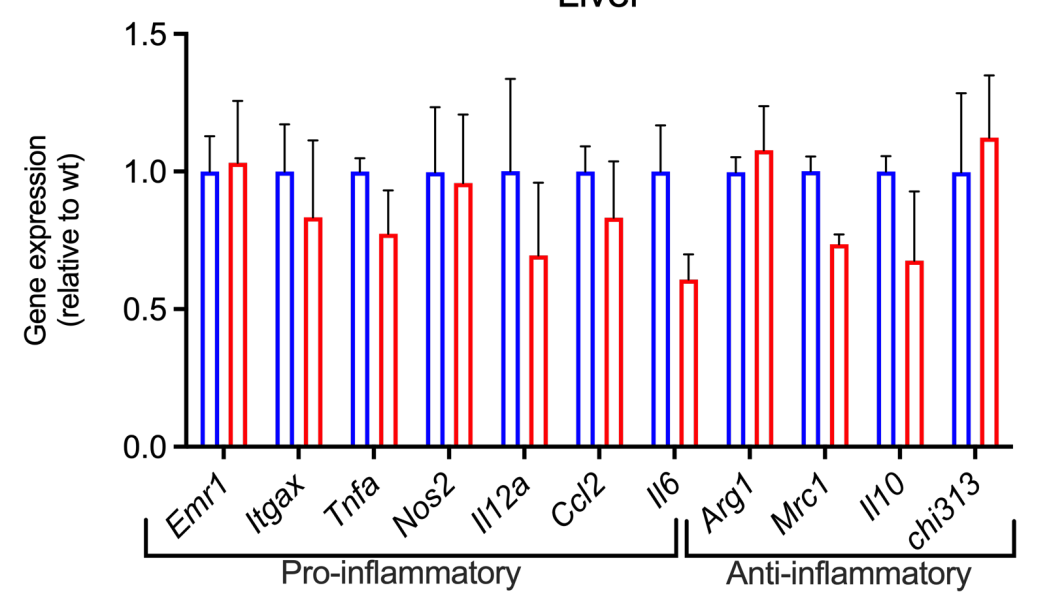

b
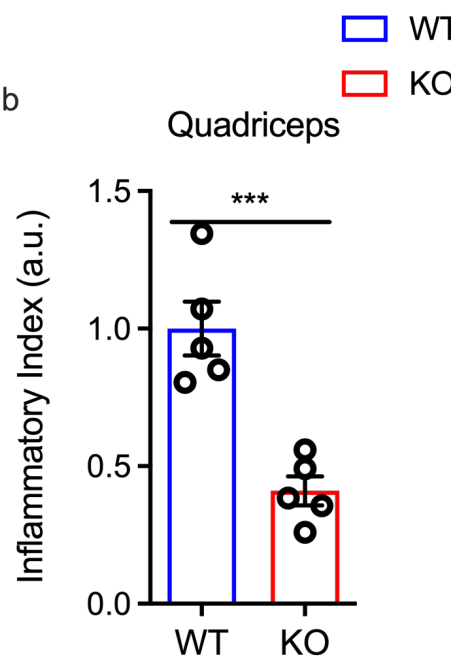

d

eWAT

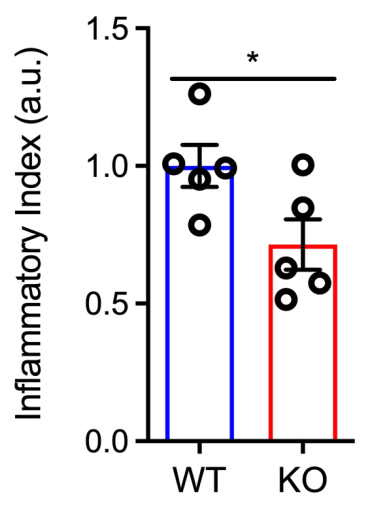

f

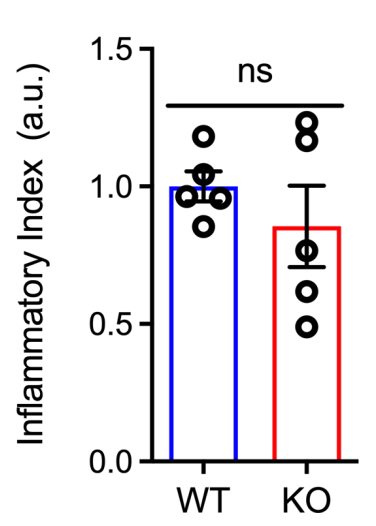




\section{Figure 7}

a

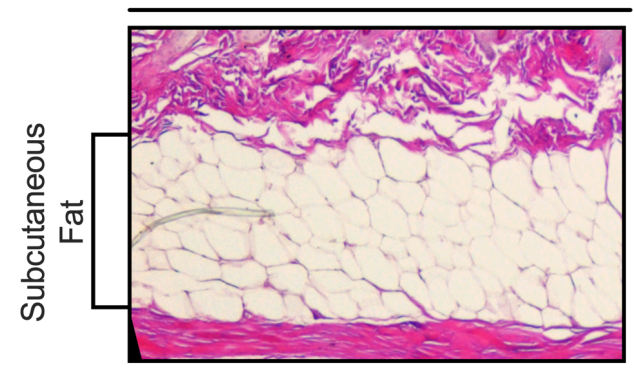

b

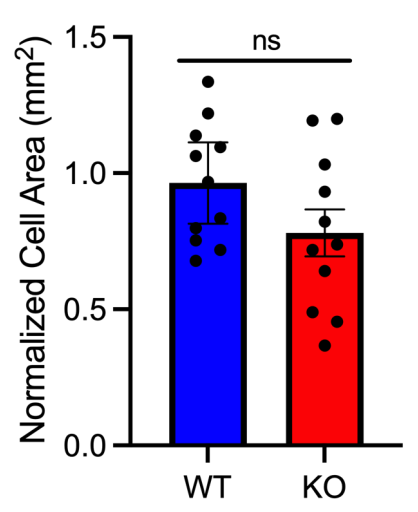

Panx3 KO

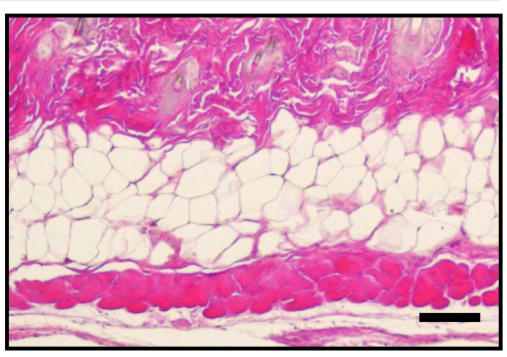

C

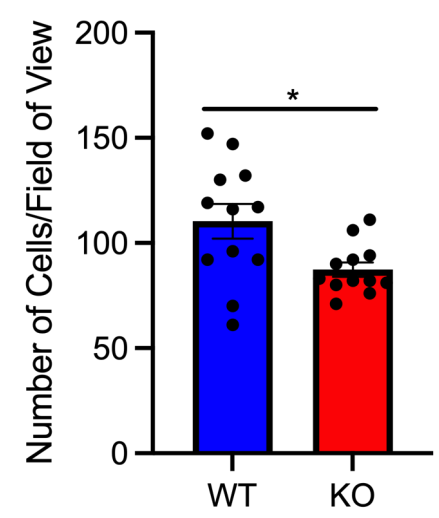

d

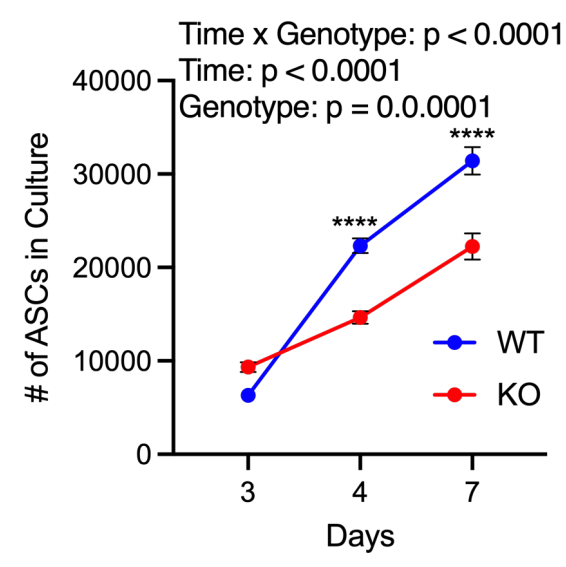

e

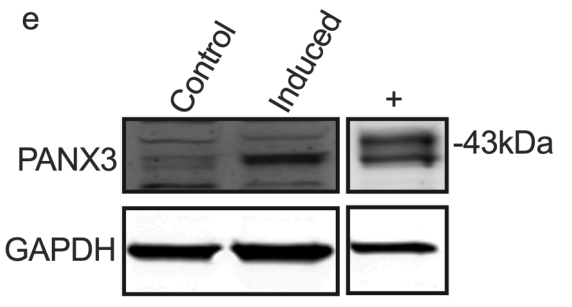

g

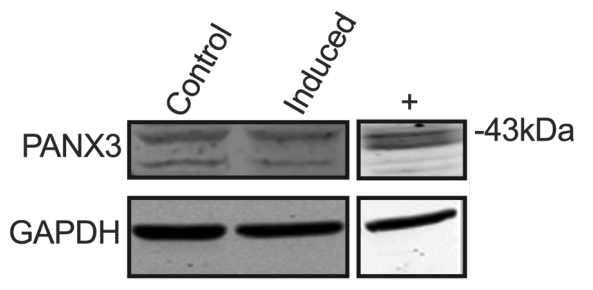

f

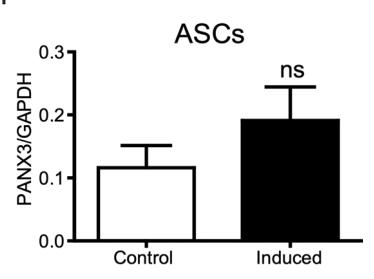

h

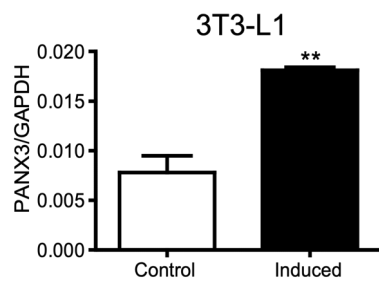

